\title{
BIRATIONAL MOTIVES, II: TRIANGULATED BIRATIONAL MOTIVES
}

\author{
BRUNO KAHN AND R. SUJATHA
}

\begin{abstract}
We develop birational versions of Voevodsky's triangulated categories of motives over a field, and relate them with the pure birational motives studied in [29]. We also get an interpretation of unramified cohomology in this framework, leading to "higher derived functors of unramified cohomology".
\end{abstract}

\section{Contents}

Introduction 1

1. Birational sheaves with transfers 4

2. Birational sheaves and pure birational motives 11

3. Triangulated birational motives 16

4. Further examples of birational sheaves 28

5. Birational sheaves and cycle modules 31

6. Unramified cohomology 34

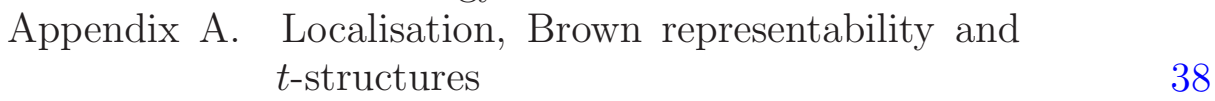

$\begin{array}{ll}\text { References } & 46\end{array}$

\section{INTRODUCTION}

This is the last part of our project on birational motives: it develops a birational analogue to Voevodsky's theory of triangulated motives. We work over a field $F$. A summary of our results may be read in the following commutative diagram of categories:

Date: April 18, 2016.

2010 Mathematics Subject Classification. 14C15, 18E30, 14E05.

The first author acknowledges the support of Agence Nationale de la Recherche (ANR) under reference ANR-12-BL01-0005 and the second author that of NSERC Grant 402071/2011. Both authors acknowledge the support of CEFIPRA project 2501-1. 


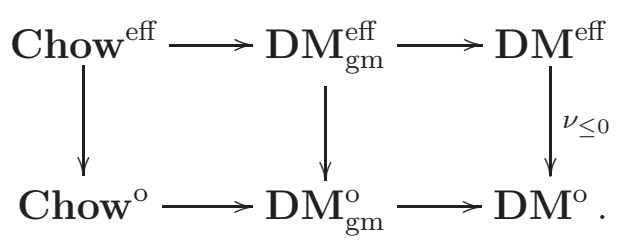

In the top row, Chow ${ }^{\text {eff }}$ is the category of effective Chow motives over $F$ (with integer coefficients), $\mathbf{D M}_{\mathrm{gm}}^{\mathrm{eff}}$ is Voevodsky's triangulated category of effective geometric motives $[53, \S 2]$ and $\mathbf{D} \mathbf{M}^{\text {eff }}$ is an unbounded version of his triangulated category of motivic complexes [53, $\S 3]$.

In the bottom row, Chow $^{\circ}$ is the category of pure birational motives introduced in [29, Def. 2.3.5]: if $X, Y$ are smooth projective varieties with motives $h^{\circ}(X), h^{\circ}(Y) \in$ Chow $^{\circ}$, we have an isomorphism $\operatorname{Hom}\left(h^{\circ}(X), h^{\circ}(Y)\right)=C H_{0}\left(Y_{F(X)}\right)$. The categories $\mathbf{D M}_{\mathrm{gm}}^{\mathrm{o}}$ and $\mathbf{D M}^{\circ}$ are respectively obtained from $\mathbf{D} \mathbf{M}_{\mathrm{gm}}^{\mathrm{eff}}$ and $\mathbf{D} \mathbf{M}^{\mathrm{eff}}$ by inverting birational morphisms (Definition 3.2.1).

When $F$ is perfect, the functors in the top row are full embeddings as a consequence of Voevodsky's main theorems on homotopy invariant pretheories [52]; by the same theorems, $\mathbf{D M}^{\text {eff }}$ enjoys a canonical "homotopy $t$-structure". All these facts turn out to be true also in the bottom row, without assuming $F$ perfect (Theorem 3.2.2); their proofs are much more elementary and don't rely on the results of [52].

The heart of the homotopy $t$-structure on $\mathbf{D M}^{\circ}$ is the category $\mathbf{H I}^{\mathrm{o}}$ of birational presheaves with transfers: these are simply the presheaves with transfers of [53, Def. 3.1.1] which invert birational morphisms. This abelian category has truly excellent properties:

- $\mathbf{H I}^{\circ}$ is a category of modules over an additive category; as such it has enough injectives, enough projectives, exact infinite direct sums and (quite unusually) exact infinite direct products ( $c f$. Proposition 1.2.1).

- A birational presheaf with transfers is automatically a Nisnevich sheaf, and is homotopy invariant; it has no higher Nisnevich cohomology (Proposition 1.3.3).

The functor $\nu_{\leq 0}$ has a right adjoint $i^{\circ}$, which in turn has a right adjoint $R_{\mathrm{nr}}$. When $F$ is perfect, one can compare the $t$-structures of $\mathrm{DM}^{\mathrm{eff}}$ and $\mathrm{DM}^{\mathrm{o}}$ : then $\nu_{\leq 0}$ is right $t$-exact, $i^{\mathrm{o}}$ is $t$-exact and $R_{\mathrm{nr}}$ is left $t$-exact. In particular, if $\mathcal{F}$ is a homotopy invariant Nisnevich sheaf with transfers, the complex $R_{\mathrm{nr}} \mathcal{F}$ is concentrated in degrees $\geq 0$. We compute its 0-cohomology sheaf $R_{\text {nr }}^{0} \mathcal{F}$ as follows: 
Theorem 1 ( $c f$. Theorem 6.3.1). For any smooth connected F-variety $X$, one has

$$
R_{\mathrm{nr}}^{0} \mathcal{F}(X)=\operatorname{Ker}\left(\mathcal{F}(K) \stackrel{\left(\partial_{v}\right)}{\longrightarrow} \prod_{v} \mathcal{F}_{-1}(F(v))\right)
$$

where $K=F(X)$ and $v$ runs through all the F-divisorial valuations on $K$. Here $\mathcal{F}_{-1}$ denotes Voevodsky's contraction of $\mathcal{F}$, cf. $\S 1.4$.

Thus we recover unramified cohomology in the sense of ColliotThélène-Ojanguren [10]: this was one of the initial aims of our project, which was not achieved in the 2002 preprint version [26]. This also shows that unramified cohomology has, in some fashion, higher derived functors which define new birational invariants: these functors are partly studied in [30].

Given the long period of gestation of this work, there have been other expositions of triangulated birational motives, notably in [20] and [24]: they are essentially independent from the present one. We would like to finish this introduction by pointing a mistake in the initial version:

In [26], Theorem 7.7, Corollary 7.8 and Corollary 7.9 c) are false: see Remark 3.6.4 below as concerns Theorem 7.7. The "upper half" of Corollary 7.9 c) remains true, as in Theorem $3.2 .2 \mathrm{e}$ ). The contents of $\S 3.7$ may be viewed as a comment on this mistake.

Acknowledgements. While writing this paper, we benefited from discussions and exchanges with a large number of colleagues. We would like to especially thank Joseph Ayoub, Alexander Beilinson, Frédéric Déglise, Eric Friedlander, Dennis Gaitsgory, Jens Hornbostel, Annette Huber-Klawitter, Bernhard Keller, Marc Levine, Georges Maltsiniotis, Fabien Morel, Amnon Neeman, Joël Riou, Raphaël Rouquier, Vladimir Voevodsky and Chuck Weibel. We also thank the referees for helpful comments.

0.1. Notation. $F$ is the base field. All varieties are $F$-varieties and all morphisms are $F$-morphisms. If $X$ is an irreducible variety, $\eta_{X}$ denotes its generic point. We write $\mathbf{S m}$ for the category of smooth varieties (= smooth separated $F$-schemes of finite type).

If $A$ is an abelian group and $p$ is a prime number, we write $A[1 / p]:=$ $A \otimes \mathbf{Z}[1 / p]$. If $\mathcal{C}$ is a category and $X, Y$ are objects of $\mathcal{C}$, we write $\mathcal{C}(X, Y)$ or $\operatorname{Hom}_{\mathcal{C}}(X, Y)$ for the set of morphisms from $X$ to $Y$, depending on which of these notations is most convenient. If $\mathcal{A}$ is an additive category, we write $\mathcal{A}^{\natural}$ for its pseudo-abelian envelope; if $p$ is a prime number, we write $\mathcal{A}[1 / p]$ for the category with the same objects and Hom groups given by $\mathcal{A}[1 / p](A, B)=\mathcal{A}(A, B)[1 / p]$. 


\section{Birational Sheaves With transfers}

In this section, we study modules over the category BFC of birational finite correspondences, which is (Definition 1.1.1) the localisation of Voevodsky's category of finite correspondences obtained by inverting birational morphisms. This category has several incarnations; elementary ones are given in Theorem 1.1.3, while less elementary ones will be given in Proposition 2.3.3 and Theorem 2.4.1. This is what gives a pivotal rôle to the category $\mathbf{H I}^{\circ}=\operatorname{Mod}-\mathbf{B F C}$ of birational presheaves with transfers. These presheaves turn out to be automatically sheaves for the Nisnevich topology, and homotopy invariant; moreover they are acyclic for Nisnevich cohomology (Proposition 1.3.3). When $F$ is perfect, they can be characterised as those homotopy invariant Nisnevich sheaves with transfers whose Voevodsky contraction vanishes (Proposition 1.5.2).

1.1. Birational finite correspondences. We start from the category SmCor introduced by Voevodsky in [53]; its objects are smooth Fvarieties and its morphisms are finite correspondences: for $X, Y \in$ $\operatorname{SmCor}, \operatorname{SmCor}(X, Y)$ is the free abelian group $c(X, Y)$ with basis the set of closed integral subschemes of $X \times Y$ which are finite and surjective over a connected component of $X$. In [36], the notation was changed from SmCor to Cor: we retain the original notation to avoid confusion with Chow correspondences between smooth projective varieties, which are also used here (see $\S 2.2$ ). In contrast to the latter, finite correspondences compose "on the nose" [36, Lemma 1.7]; together with the product of varieties and cycles, they make SmCor an additive $\otimes$-category. The "graph" functor $\mathbf{S m} \rightarrow$ SmCor is the identity on objects and sends a morphism to its graph.

1.1.1. Definition. The category of birational finite correspondences is $\mathrm{BFC}=S_{b}^{-1} \mathbf{S m C o r}$, where $S_{b}$ is the class of (graphs of) birational morphisms. ${ }^{1}$

We note that $S_{b}$ is closed under disjoint unions and products; hence, by [29, Prop. A.1.2 and Th. A.3.1 ], the additive and tensor structures of SmCor pass to BFC.

We shall use other incarnations of BFC. It is helpful to use the category of [36, Def. 2.25]: its objects are smooth varieties and morphisms

\footnotetext{
${ }^{1}$ More correctly, a morphism $f: X \rightarrow Y$ is in $S_{b}$ if it is dominant and its restriction to any connected component of $X$ yields a birational morphism to some connected component of $Y$; we would get the same localisation by using the class $S_{\mathrm{o}}$ of dense open immersions. See $[28,1.7]$ and $[29,2.1]$ for detailed discussions.
} 
between two smooth varieties $X, Y$ are given by

$$
h_{0}(X, Y)=\operatorname{Coker}\left(c\left(X \times \mathbf{A}^{1}, Y\right) \stackrel{i_{1}(X)^{*}-i_{0}(X)^{*}}{\longrightarrow} c(X, Y)\right)
$$

where $i_{t}: \operatorname{Spec} k \rightarrow \mathbf{A}^{1}$ is the inclusion of the point $t$ and $i_{t}(X)=$ $i_{t} \times 1_{X}$. We denote this category by $\mathcal{H}(\mathrm{SmCor})$.

1.1.2. Lemma. Let $S_{h}$ be the class of projections $\pi_{X}: X \times \mathbf{A}^{1} \rightarrow X$. Then $\mathcal{H}(\mathbf{S m C o r})$ is isomorphic to $S_{h}^{-1} \mathbf{S m C o r}$.

Proof. Let us show that $\mathcal{H}(\mathbf{S m C o r})$ and $S_{h}^{-1}$ SmCor have each other's universal property. Let $F:$ SmCor $\rightarrow \mathcal{C}$ be a functor. If $F$ factors through $S_{h}^{-1}$ SmCor, then $F\left(i_{0}(X)\right)=F\left(i_{1}(X)\right)$ for any $X$, as both are inverse to $F\left(\pi_{X}\right)$. Hence $F$ factors through $\mathcal{H}$ (SmCor). On the other hand, $\pi_{X}$ is invertible in $\mathcal{H}$ (SmCor) (see comment after [36, Def. 2.25]). Hence, if $F$ factors through $\mathcal{H}(\mathrm{SmCor})$, it also factors through $S_{h}^{-1}$ SmCor.

1.1.3. Theorem. In the diagram

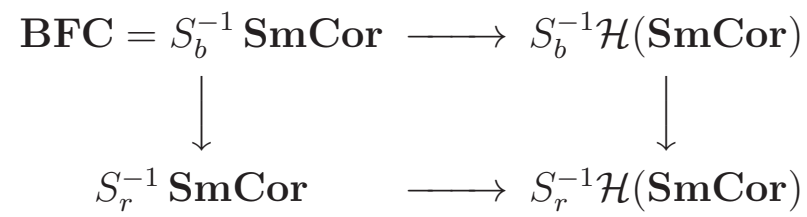

all functors are isomorphisms of categories. Here $S_{r}$ denotes the class of [graphs of] stable birational morphisms. ${ }^{2}$

Proof. It follows from Lemma 1.1.2 and [28, Lemma 1.7.1] that the bottom horizontal functor is an isomorphism of categories; on the other hand, the vertical functors are isomorphisms of categories by $[28, \mathrm{Th}$. 1.7.2].

We shall get further descriptions of BFC in Theorem 2.4.1 and Remark 2.4.3.

1.2. Review of modules over additive categories. We refer to [1, $\S 1$ and [31, App. A] for this additive version of [SGA4-I, I.5.3]. Let $\mathcal{A}$ be an (essentially small) additive category: we recall the fully faithful additive Yoneda functor

$$
\mathcal{A} \stackrel{y}{\longrightarrow} \operatorname{Mod}-\mathcal{A}
$$

where $\operatorname{Mod}-\mathcal{A}$ is the category of right $\mathcal{A}$-modules (= contravariant additive functors from $\mathcal{A}$ to abelian groups). Let us call an object

\footnotetext{
${ }^{2} \mathrm{~A}$ morphism between connected smooth varieties is stably birational if it is dominant and induces a purely transcendental extension of function fields; this is extended to nonconnected smooth varieties as in footnote 1.
} 
of $\operatorname{Mod}-\mathcal{A}$ representable if it is in the image of the Yoneda functor $y: \mathcal{A} \rightarrow \operatorname{Mod}-\mathcal{A}$ and free if it is a direct sum of representable objects. Also recall that an object $X$ of a category $\mathcal{C}$ is compact if $\mathcal{C}(X,-)$ commutes with arbitrary direct limits (representable in $\mathcal{C}$ ). We then have the following general facts ([1, Prop. 1.3.6 and A.1.4], see also [SGA4-I, Exp. 1, p. 97, Ex. 8.7.8] in the nonadditive case):

1.2.1. Proposition. a) The category $\operatorname{Mod}-\mathcal{A}$ is abelian, has enough projectives and enough injectives and admits arbitrary direct and inverse limits. Filtering direct limits and products are exact.

b) Let $\mathcal{A}^{\natural}$ be the pseudo-abelian envelope of $\mathcal{A}$; then $y$ extends to a full embedding $\mathcal{A}^{\natural} \hookrightarrow \operatorname{Mod}-\mathcal{A}$. Its image consists of all compact objects, and these objects are projective.

c) Any free object is projective and there are enough free objects.

If $f: \mathcal{A} \rightarrow \mathcal{B}$ is an additive functor, it induces a triple of adjoint functors, with $\left(f^{*} \mathcal{F}\right)(A)=\mathcal{F}(f(A))$ for $\left.\mathcal{F} \in \operatorname{Mod}-\mathcal{B}\right)$ :

$$
\operatorname{Mod}-\mathcal{A} \underset{\stackrel{f_{*}}{\stackrel{f^{*}}{\longrightarrow}}}{\stackrel{f_{!}}{\longrightarrow}} \operatorname{Mod}-\mathcal{B}
$$

(each functor is left adjoint to the one below it), and $f_{\text {! naturally com- }}$ mutes with $f$ relatively to the Yoneda embeddings. If $\mathcal{F} \in \operatorname{Mod}-\mathcal{A}$, the unit (resp. counit) morphism

$$
\mathcal{F} \stackrel{\eta}{\longrightarrow} f^{*} f_{!} \mathcal{F} \quad\left(\text { resp. } f^{*} f_{*} \mathcal{F} \stackrel{\varepsilon}{\longrightarrow} \mathcal{F}\right)
$$

is then universal among morphisms from $\mathcal{F}$ to (resp. to $\mathcal{F}$ from) presheaves of the form $f^{*} \mathcal{G}$. Note that $f^{*}$, hence $f_{\text {! }}$, is an equivalence of categories for $f: \mathcal{A} \rightarrow \mathcal{A}^{\natural}$ the canonical embedding.

The functor $f^{*}$ is fully faithful in two cases: when $f$ is a localisation, or when $f$ is full and essentially surjective. These facts are left to the readers as exercises. This persists if one further passes to pseudoabelian envelopes.

If $\mathcal{A}$ is monoidal, its tensor structure extends to $\operatorname{Mod}-\mathcal{A}$ in such a way that the Yoneda embedding is monoidal; if $f: \mathcal{A} \rightarrow \mathcal{B}$ is a monoidal functor between (additive) monoidal categories, then $f_{\text {! }}$ is monoidal [31, A.8, A.12].

1.3. Elementary properties of birational sheaves. With the notation of $\S 1.2$, the category PST of presheaves with transfers of [53, Def. 3.1.1] or [36, Def. 2.1] is none other than Mod-SmCor.

1.3.1. Definition. We denote by $\mathbf{H I}^{\mathrm{O}}$ the full subcategory of $\mathbf{P S T}$ consisting of those presheaves $\mathcal{F}$ that are birationally invariant, i.e. 
such that $\mathcal{F}(X) \stackrel{\sim}{\longrightarrow} \mathcal{F}(U)$ for any dense open immersion $j: U \rightarrow X$. We call an object of $\mathbf{H I}^{\circ}$ a birationally invariant homotopy invariant presheaf with transfers, or for short, a birational sheaf [with transfers].

By definition, the obvious functor

$$
\mathbf{H I}^{\mathrm{O}} \rightarrow \mathrm{Mod}-\mathbf{B F C}=\operatorname{Mod}-\mathbf{B F C}^{\natural}
$$

is an isomorphism of categories; Proposition 1.2.1 therefore applies.

Let PHI denote the full subcategory of PST consisting of homotopy invariant presheaves with transfers. We may identify PHI with Mod- $\mathcal{H}($ SmCor) (see $\S 1.1)$. In view of Proposition 1.2.1 a), the string of functors $\mathrm{SmCor} \stackrel{\alpha}{\longrightarrow} \mathcal{H}(\mathrm{SmCor}) \stackrel{\beta}{\longrightarrow}$ BFC yields a naturally commutative diagram of categories (ibid.)

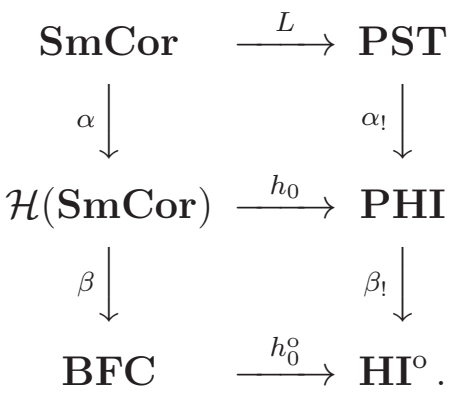

Here we follow the notation of [53, p. 199 and 207] for the two top Yoneda functors (in [36], the notation $L$ is replaced by $\mathbf{Z}_{t r}$ ): for $Y \in \mathbf{S m}, h_{0}(Y)$ is the presheaf $X \mapsto h_{0}(X, Y)$ (cf. (1.1)). As in [53], we also write $\alpha_{!} \mathcal{F}=h_{0}(\mathcal{F})$ for $\mathcal{F} \in \mathbf{P S T}$. We will sometimes write $\mathcal{F}^{\circ}=\beta_{!} \alpha_{!} \mathcal{F}$, so that the canonical map $\mathcal{F} \rightarrow \alpha^{*} \beta^{*} \mathcal{F}^{\circ}$ is universal among morphisms from $\mathcal{F}$ to birational presheaves. If $\mathcal{F} \in \mathbf{P H I}$, we have

$$
\mathcal{F}^{\mathrm{o}}=\beta_{!} \alpha_{!} \alpha^{*} \mathcal{F}=\beta_{!} \mathcal{F} \text {. }
$$

We shall also use the category of Nisnevich sheaves with transfers [53, Def. 3.1.1], [36, Lect. 13], that we denote here by NST: by definition, this is the full subcategory of PST formed by those presheaves with transfers which are sheaves in the Nisnevich topology. We also write $\mathbf{H I}=\mathbf{N S T} \cap \mathbf{P H I}$ for the category of homotopy invariant Nisnevich sheaves with transfers (see [53, Prop. 3.1.13]). Recall the exact sheafification functor [53, Th. 3.1.4]

$$
a: \mathrm{PST} \rightarrow \mathrm{NST}
$$

which is left adjoint of the inclusion functor $k: \mathbf{N S T} \hookrightarrow \mathbf{P S T}$.

The following innocent-looking lemma turns out to be very powerful, and justifies the terminology "birational sheaf". 
1.3.2. Lemma. a) Any presheaf of sets $\mathcal{F}$ on $\mathbf{S m}$ which transforms coproducts into products and is birationally invariant in the sense that $\mathcal{F}(X) \stackrel{\sim}{\longrightarrow} \mathcal{F}(U)$ for any dense open immersion $U \hookrightarrow X$ is a sheaf for the Nisnevich topology.

b) If $\mathcal{F}$ is moreover a (pre) sheaf of abelian groups, then $H_{\mathrm{Nis}}^{i}(X, \mathcal{F})=0$ for all $X \in \mathbf{S m}$ and all $i \neq 0$.

Proof. a) This follows from [40, p. 96, Prop. 1.4]. b) This follows from [44, Lemma 1.40].

1.3.3. Proposition. a) One has $\mathbf{H I}^{\mathrm{O}} \subset \mathbf{H I}$.

b) For any $\mathcal{F} \in \mathbf{H I}^{\mathbf{O}}$ and any $X \in \mathbf{S m}$, one has $H_{\mathrm{Nis}}^{i}(X, \mathcal{F})=0$ for all $i \neq 0$.

Proof. a) Let $\mathcal{F} \in \mathbf{H I}^{\circ}$. By Lemma 1.3.2 a), $\mathcal{F}$ is a sheaf in the Nisnevich topology. The fact that it is homotopy invariant follows from [28, Th. 1.7.2]. b) merely repeats Lemma 1.3 .2 b). ${ }^{3}$

1.4. Contractions. Recall the following definition from [52, p. 96] or [36, Lect. 23]:

1.4.1. Definition. Let $\mathcal{F} \in \mathbf{P S T}$. Then $\mathcal{F}_{-1} \in \mathbf{P S T}$ is the presheaf with transfers defined by

$$
\mathcal{F}_{-1}(X)=\operatorname{Coker}\left(\mathcal{F}\left(X \times \mathbf{A}^{1}\right) \rightarrow \mathcal{F}\left(X \times\left(\mathbf{A}^{1}-\{0\}\right)\right)\right) .
$$

This is the contraction of $\mathcal{F}$.

Note that if $\mathcal{F} \in \mathbf{P H I}, \mathcal{F}_{-1}(X)$ is a functorial direct summand of $\mathcal{F}\left(X \times\left(\mathbf{A}^{1}-\{0\}\right)\right)$ because the map $\mathcal{F}(X) \rightarrow \mathcal{F}\left(X \times\left(\mathbf{A}^{1}-\{0\}\right)\right)$ has a section given by $1 \in \mathbf{A}^{1}-\{0\}$; in particular, $\mathcal{F}_{-1} \in \mathbf{P H I}$. If $\mathcal{F} \in \mathbf{H I}$, this argument shows that $\mathcal{F}_{-1} \in \mathbf{H I}$, hence $\mathcal{F} \mapsto \mathcal{F}_{-1}$ defines an exact endofunctor of $\mathbf{H I}$.

We may extend $\mathcal{F}$ to smooth separated $F$-schemes essentially of finite type by taking direct limits over open sets, in a standard way; in particular, we write $\mathcal{F}(K)$ for $\mathcal{F}(\operatorname{Spec} K)=\lim _{\longrightarrow} \mathcal{F}(U)$ if $K$ is the function field of a smooth irreducible variety $X \overrightarrow{\text { and }} U$ runs through its open subsets. Recall the following theorem of Voevodsky, a special case of his Gersten resolution [52, Th. 4.37]:

1.4.2. Theorem. Let $F$ be perfect and suppose $\mathcal{F} \in \mathbf{H I}$. Then there is an exact sequence for any $X \in \mathbf{S m}$ :

$$
0 \rightarrow \mathcal{F}(X) \rightarrow \mathcal{F}(F(X)) \stackrel{\left(\partial_{x}\right)}{\longrightarrow} \bigoplus_{x \in X^{(1)}} \mathcal{F}_{-1}(F(x))
$$

\footnotetext{
${ }^{3}$ In particular it is not necessary to invoke [53, Th. 3.1.12] as we did in [26]: we owe this remark to Joël Riou.
} 
Here $\partial_{x}$ is defined from the purity isomorphism of ibid., Lemma 4.36.

1.5. Further characterisations of birational sheaves. The following characterisations are sometimes useful: they assume $F$ to be perfect. The first one is extracted from [31, Lemma $10.3 \mathrm{~b})]$ :

1.5.1. Proposition. Assume $F$ perfect, and let $\mathcal{F} \in \mathbf{H I}$. Then $\mathcal{F} \in$ $\mathbf{H I}^{\mathrm{O}}$ if and only if the following holds:

For any function field $K / F$, for any regular curve $C$ over $K$ and any closed point $c \in C$, the induced map $\mathcal{F}\left(\mathcal{O}_{C, c}\right) \rightarrow \mathcal{F}(K(C))$ is surjective.

(These sheaves are called universally proper in [31].)

The second one uses the notion of contraction that we just recalled:

1.5.2. Proposition. Let $\mathcal{F} \in \mathbf{H I}$. Consider the following conditions:

(i) $\mathcal{F} \in \mathbf{H I}^{\circ}$.

(ii) $\mathcal{F}(X) \stackrel{\sim}{\longrightarrow} \mathcal{F}\left(X \times\left(\mathbf{A}^{1}-\{0\}\right)\right)$ for any $X \in \mathbf{S m}$.

(iii) $\mathcal{F}_{-1}=0$.

Then (i) $\Rightarrow$ (ii) $\Longleftrightarrow$ (iii); if F is perfect, (iii) $\Rightarrow$ (i).

Proof. The first implications are obvious; the last one follows from Theorem 1.4.2.

1.6. Serre embeddings. Let $\mathcal{A}$ be an abelian category. Recall that a full subcategory $\mathcal{B} \subseteq \mathcal{A}$ is a Serre subcategory if it is additive and if, given an exact sequence $0 \rightarrow A^{\prime} \rightarrow A \rightarrow A^{\prime \prime} \rightarrow 0$ in $\mathcal{A}$, we have $A \in \mathcal{B}$ $\Longleftrightarrow A^{\prime}, A^{\prime \prime} \in \mathcal{B}$. We say that $\mathcal{B} \rightarrow \mathcal{A}$ is a Serre embedding. We have:

1.6.1. Proposition. let $f^{*}: \mathcal{B} \rightarrow \mathcal{A}$ be a Serre embedding. Suppose that $f^{*}$ has a left adjoint $f_{!}$(resp. a right adjoint $f_{*}$ ). Then, for any $\mathcal{F} \in \mathcal{A}$ the unit (resp. counit) morphism of (1.3) is an epimorphism (resp. a monomorphism).

Proof. By duality, it suffices to prove this for $f_{*}$. This is [3, Prop. E.4.1 (1)], whose proof we reproduce here for completeness. Since in particular $f^{*}$ is fully faithful, the unit morphism $\mathcal{G} \rightarrow f_{*} f^{*} \mathcal{G}$ is an isomorphism for any $\mathcal{G} \in \mathcal{B}$. Let $\mathcal{F} \in \mathcal{A}$ and let $C=\operatorname{Ker}\left(f^{*} f_{*} \mathcal{F} \rightarrow \mathcal{F}\right)$ : by hypothesis, $C \simeq f^{*} C^{\prime}$ for some $C^{\prime} \in \mathcal{B}$. Applying the left exact functor $f_{*}$ to the exact sequence $0 \rightarrow C \rightarrow f^{*} f_{*} \mathcal{F} \rightarrow \mathcal{F}$ yields a diagram

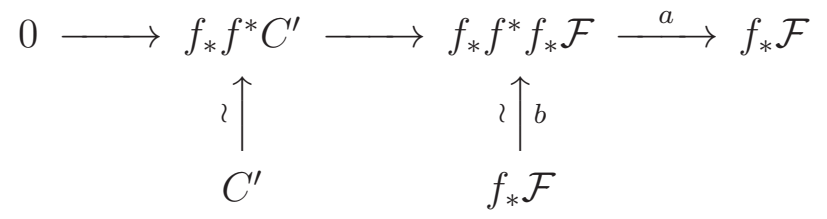


in which $a b=1_{f_{*} \mathcal{F}}$ by the adjunction identities. Hence in the top exact row, $a$ is an isomorphism and $f_{*} f^{*} C^{\prime}=0$.

For the sequel it is important to know that some inclusions of abelian subcategories of PST are Serre embeddings. We treat all of them in a unified way.

1.6.2. Proposition. Suppose F perfect. Then the inclusions $\iota: \mathbf{P H I}$ $\subset \mathbf{P S T}, i: \mathbf{H I} \subset \mathbf{N S T}$ and $i^{\mathrm{O}}: \mathbf{H I}^{\mathrm{O}} \subset \mathbf{H I}$ are Serre embeddings.

Proof. Let $\mathcal{F} \in$ PST. For any smooth variety $X$, the map

$$
\mathcal{F}(X) \rightarrow \mathcal{F}\left(X \times \mathbf{A}^{1}\right)
$$

is split by using the rational point $0 \in \mathbf{A}^{1}$. This defines an idempotent endomorphism of $\mathcal{F}\left(X \times \mathbf{A}^{1}\right)$, whose kernel we denote by $\tilde{\mathcal{F}}(X)$ : this is a presheaf in $X$. So $\mathcal{F} \in \mathbf{P H I} \Longleftrightarrow \tilde{\mathcal{F}}=0$.

The construction $\mathcal{F} \mapsto \tilde{\mathcal{F}}$ is clearly functorial in $\mathcal{F}$, and exact. In particular, if $0 \rightarrow \mathcal{F}^{\prime} \rightarrow \mathcal{F} \rightarrow \mathcal{F}^{\prime \prime} \rightarrow 0$ is a short exact sequence in PST and $X \in \mathbf{S m C o r}$, we get a short exact sequence

$$
0 \rightarrow \tilde{\mathcal{F}}^{\prime}(X) \rightarrow \tilde{\mathcal{F}}(X) \rightarrow \tilde{\mathcal{F}}^{\prime \prime}(X) \rightarrow 0 .
$$

That $\iota$ is a Serre embedding follows immediately. (In this case, the perfectness of $F$ is not used.)

Suppose now that $0 \rightarrow \mathcal{F}^{\prime} \rightarrow \mathcal{F} \rightarrow \mathcal{F}^{\prime \prime} \rightarrow 0$ is an exact sequence in NST. Given $X \in \mathbf{S m C o r}$, the cohomology exact sequence induces an exact sequence

$$
0 \rightarrow \tilde{\mathcal{F}}^{\prime}(X) \rightarrow \tilde{\mathcal{F}}(X) \rightarrow \tilde{\mathcal{F}}^{\prime \prime}(X) \rightarrow \tilde{H}_{\text {Nis }}^{1}\left(X, \mathcal{F}^{\prime}\right) .
$$

(Here we viewed $\mathcal{G}=H_{\mathrm{Nis}}^{1}\left(-, \mathcal{F}^{\prime}\right)$ as a presheaf on $\mathbf{S m}$, and wrote $\tilde{H}_{\text {Nis }}^{1}\left(X, \mathcal{F}^{\prime}\right)$ for $\tilde{\mathcal{G}}(X)$.) This shows that $\mathbf{H I}$ is closed under extensions in NST. Conversely, suppose that $\mathcal{F} \in \mathbf{H I}$. Then $\mathcal{F}^{\prime} \in \mathbf{H I}$. But a theorem of Voevodsky [53, Th. 3.1.12] implies that $X \mapsto H_{\mathrm{Nis}}^{1}\left(X, \mathcal{F}^{\prime}\right)$ is homotopy invariant, hence $\mathcal{F}^{\prime \prime} \in \mathbf{H I}$ as well.

To deal with the last case, we argue as above using this time $\mathbf{A}^{1}-\{0\}$. Thus, for $\mathcal{F} \in$ PST, write $\check{\mathcal{F}}(X)$ for the kernel of the idempotent on $\mathcal{F}\left(X \times\left(\mathbf{A}^{1}-\{0\}\right)\right)$ defined by the rational point $1 \in \mathbf{A}^{1}-\{0\}$. Suppose $\mathcal{F} \in \mathbf{H I}$ : by Proposition 1.5.2, $\mathcal{F} \in \mathbf{H I}^{\circ} \Longleftrightarrow \check{\mathcal{F}}=0$.

Suppose that $0 \rightarrow \mathcal{F}^{\prime} \rightarrow \mathcal{F} \rightarrow \mathcal{F}^{\prime \prime} \rightarrow 0$ is an exact sequence in $\mathbf{H I}$. For any $X \in \mathbf{S m}$, we have an exact sequence

$$
0 \rightarrow \check{\mathcal{F}}^{\prime}(X) \rightarrow \check{\mathcal{F}}(X) \rightarrow \check{\mathcal{F}}^{\prime \prime}(X) \rightarrow \check{H}_{\text {Nis }}^{1}\left(X, \mathcal{F}^{\prime}\right) .
$$

This exact sequence shows that $\mathbf{H I}^{\mathrm{O}}$ is closed under extensions in HI. Now suppose that $\mathcal{F} \in \mathbf{H I}^{\circ}$. Then $\mathcal{F}^{\prime} \in \mathbf{H I}^{\circ}$. But lemma 1.3.2 
b) implies that $H_{\mathrm{Nis}}^{1}\left(X, \mathcal{F}^{\prime}\right)=0$, and a fortiori $\check{H}_{\mathrm{Nis}}^{1}\left(X, \mathcal{F}^{\prime}\right)=0$. So $\mathcal{F}^{\prime \prime} \in \mathbf{H I}^{\mathrm{O}}$ as well.

Recall that in Proposition 1.6.2, $i$ has a left adjoint: this nontrivial fact amounts to say that $a(\mathbf{P H I}) \subseteq \mathbf{H I}[53$, Prop. 3.1.12]. More easily:

1.6.3. Proposition. In Proposition 1.6.2, $i^{\mathrm{o}}$ has a left adnoint $\nu_{0}$ and a right adjoint $R_{\mathrm{nr}}^{0}$. If $F$ is perfect, the canonical morphism $\mathcal{F} \rightarrow \nu_{0} \mathcal{F}$ (resp. $R_{\mathrm{nr}}^{0} \mathcal{F} \rightarrow \mathcal{F}$ ) is an epimorphism (resp. a monomorphism) for any $\mathcal{F} \in \mathbf{H I}$.

Proof. Since $\mathbf{H I}^{\mathrm{O}} \subset \mathbf{H I}$, it is clear with the notation of (1.4) that the composition $\mathbf{H I} \hookrightarrow \mathbf{P H I} \stackrel{\beta_{!}}{\longrightarrow} \mathbf{H I}^{\mathrm{o}}$ (resp. $\mathbf{H I} \hookrightarrow \mathbf{P H I} \stackrel{\beta_{*}}{\longrightarrow} \mathbf{H I}^{\mathrm{o}}$ ) yields the desired left (resp. right) adjoint (see also Proposition 3.3.6). The last two claims now follow from Propositions 1.6.1 and 1.6.2.

\section{Birational SHEAVES AND PURE BIRATIONAL MOTIVES}

We construct in Proposition 2.3.3 a full embedding of the category Cor $_{\text {rat }}^{\circ}$ of birational Chow correspondences, introduced in [29], into the category BFC studied in the previous section. This relies on an explicit formula for the Nisnevich sheaf with transfers $h_{0}^{\mathrm{Nis}}(Y)$ attached to a smooth proper variety $Y$, which imples that it is birational (Theorem 2.1.2); when $F$ is perfect, this was proven differently in [20, Proof of Th. 2.2]. The full embedding $\mathbf{C o r}_{\text {rat }}^{\circ} \hookrightarrow \mathbf{B F C}$ becomes an equivalence of categories after inverting the exponential characteristic and passing to idempotent completions (Corollary 2.3.4). As a first byproduct, we get in Corollary 2.4.2 a functor

$$
S_{b}^{-1} \mathbf{S m} \rightarrow \text { Chow }^{\mathrm{o}}[1 / p]
$$

where $S_{b}^{-1} \mathbf{S m}$ is the category which was studied in [28] and $\mathbf{C h o w}^{\mathrm{o}}$ is the category of birational Chow motives which was studied in [29]; this functor could not be construced by the methods of [29].

\subsection{Birational sheaves and smooth projective varieties.}

2.1.1. Lemma. Let $X, Y \in \mathbf{S m}$. Then we have a natural homomorphism (cf. (1.1))

$$
h_{0}(X, Y) \rightarrow C H^{\operatorname{dim} Y}(X \times Y)
$$

which is bijective if $X=\operatorname{Spec} F$ and $Y$ is proper. 
Proof. The first claim is obvious from the commutative diagram

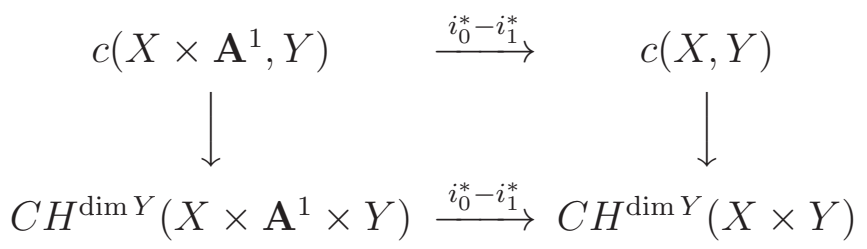

and the homotopy invariance of Chow groups. Suppose that $X=$ Spec $F$. Then $c(X, Y)=Z_{0}(Y)$; if $Y$ is proper, any irreducible 1-cycle on $\mathbf{A}^{1} \times Y$ which is not constant over $\mathbf{A}^{1}$ defines an element of $c\left(\mathbf{A}^{1}, Y\right)$, hence the second claim.

Let $Y$ be a smooth variety. As in [53, p. 207] we write $h_{0}^{\mathrm{Nis}}(Y)$ for the Nisnevich sheaf with transfers associated to the presheaf $h_{0}(Y) \in \mathbf{P H I}$ (see (1.4)). Note that $h_{0}^{\mathrm{Nis}}(Y) \in \mathbf{H I}$ by [53, Th. 3.1.12].

2.1.2. Theorem. Let $Y$ be a smooth proper variety. Then $h_{0}^{\mathrm{Nis}}(Y)$ is given by the formula

$$
h_{0}^{\mathrm{Nis}}(Y)(X)=C H_{0}\left(Y_{F(X)}\right)
$$

for any connected $X \in \mathbf{S m}$. In particular, $h_{0}^{\mathrm{Nis}}(Y) \in \mathbf{H I}^{\mathrm{O}}$.

Proof. For $X$ smooth, consider the composition

$$
c(X, Y) \rightarrow C H^{\operatorname{dim} Y}(X \times Y) \rightarrow C H^{\operatorname{dim} Y}\left(Y_{F(X)}\right)=C H_{0}\left(Y_{F(X)}\right) .
$$

Let $\alpha \in c(Z, X)$ be a finite correspondence, with $Z$ connected. We claim that the usual composition of correspondences yields a commutative diagram

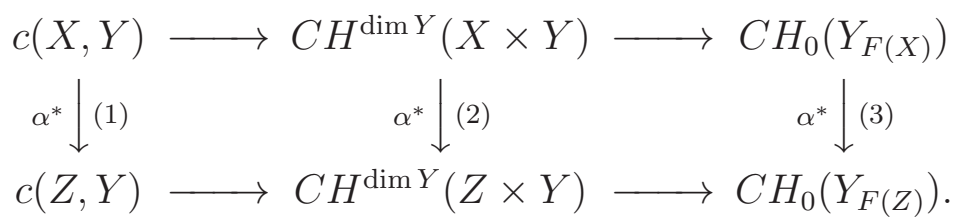

Indeed, $\alpha^{*}$ is well-defined at (2) because $Y$ is proper and the components of $\alpha$ are proper over $Z$. It obviously commutes with (1).

For (3), we must show that if $W \in Z_{\operatorname{dim} X}(X \times Y)$ is supported on a proper closed subset $X^{\prime}$ of $X$, then $\alpha^{*} W$ goes to 0 in $C H_{0}\left(Y_{F(Z)}\right)$. We argue as in the proof of [29, Prop. 2.3.4]: by passing to the generic point of $Z$ and by base change, we reduce to the case where $Z=\operatorname{Spec} F$. Then $\alpha$ is a 0 -cycle on $X$, that we may assume to be a closed point. Shrinking around $\alpha$, we may also assume $X$ to be quasi-projective.

If $\alpha \notin X^{\prime}$, then $\alpha \cap W=\emptyset$ and we are done; otherwise we may move $\alpha$ outside $X^{\prime}$ up to rational equivalence [45]: this does not change the value of $\alpha^{*} W$ in $C H_{0}(Y)$. 
Thus we have defined a presheaf with transfers

$$
\bar{h}_{0}(Y): X \mapsto C H_{0}\left(Y_{F(X)}\right)
$$

which is clearly birationally invariant. Hence, by Proposition 1.3.3 a), $\bar{h}_{0}(Y) \in \mathbf{H I}^{\mathrm{o}}$, and the morphism $L(Y) \rightarrow \bar{h}_{0}(Y)$ described in $(2.2)$ induces a morphism

$$
\varphi: h_{0}^{\mathrm{Nis}}(Y) \rightarrow \bar{h}_{0}(Y)
$$

in $\mathbf{H I}$.

To prove that $\varphi$ is an isomorphism, it is sufficient by [52, Prop. 4.20] to check that it is an isomorphism at Spec $K$ for any extension $K$ of $F$. Noting that

$$
\begin{gathered}
h_{0}^{\mathrm{Nis}}(Y)(\operatorname{Spec} K)=h_{0}(Y)(\operatorname{Spec} K)=h_{0}\left(Y_{K}\right)(\operatorname{Spec} K), \\
\bar{h}_{0}(Y)(\operatorname{Spec} K)=\bar{h}_{0}\left(Y_{K}\right)(\operatorname{Spec} K),
\end{gathered}
$$

the statement follows from Lemma 2.1.1, where $F$ is replaced by $K$ and $Y$ by $Y_{K}$.

A first consequence is:

2.1.3. Corollary. Let $Y$ be a smooth proper variety viewed as an object of $\mathbf{B F C}$. Then the associated presheaf of abelian groups $h_{0}^{\circ}(Y) \in \mathbf{H I}^{\mathrm{o}}$ (compare Diagram (1.4)) is canonically isomorphic to $h_{0}^{\mathrm{Nis}}(Y)$. In particular, $h_{0}^{\mathrm{Nis}}(Y)$ is a projective object of $\mathbf{H I}^{\mathrm{O}}$.

Proof. By definition, $h_{0}^{\mathrm{Nis}}(Y)$ is the Nisnevich sheaf associated to the presheaf $h_{0}(Y)$. Let $\mathcal{F} \in \mathbf{H I}^{\circ}$. By Lemma 1.3 .2 a), $\mathcal{F}$ is a Nisnevich sheaf, hence any map $h_{0}(Y) \rightarrow \mathcal{F}$ factors uniquely through $h_{0}^{\mathrm{Nis}}(Y)$. Since the latter is birational by Theorem $2.1 .2, h_{0}^{\mathrm{Nis}}(Y)$ has the same universal property as $h_{0}^{\circ}(Y)$, so they coincide. The last statement follows from Proposition 1.2.1 b).

2.2. Review of some results of [29]. Let Cor $_{\text {rat }}$ and Chow ${ }^{\text {eff }}$ denote respectively the category of Chow correspondences and of effective Chow motives over $F$, with integral coefficients, so that by definition Chow $^{\text {eff }}=$ Cor $_{\text {rat }}^{\natural}$. In [29, Def. 2.2.6 and 2.3.5], we defined two new categories:

$$
\text { Chow }^{\mathrm{b}}=\left(\text { Chow }^{\text {eff }} / \mathcal{L}\right)^{\natural}, \quad \text { Chow }^{\mathrm{o}}=\left(\operatorname{Cor}_{\text {rat }} / \mathcal{I}\right)^{\natural} .
$$

Here $\mathcal{L}$ is the $\otimes$-ideal generated by the Lefschetz motive $\mathbb{L}$ while, for two smooth projective varieties $X, Y, \mathcal{I}(X, Y)$ is the subgroup of $C H_{\operatorname{dim} X}(X \times Y)$ generated by correspondences with support in $Z \times Y$ 
for some proper closed subset $Z \subset X$. Writing $\mathbf{C o r}_{\text {rat }}^{\circ}=\mathbf{C o r}_{\text {rat }} / \mathcal{I}$, Hom groups in $\mathbf{C o r}_{\text {rat }}^{\mathrm{o}}$ are given by the formula [29, Lemma 2.3.6]

$$
\operatorname{Cor}_{\text {rat }}^{\mathrm{o}}(X, Y)=C H_{0}\left(Y_{F(X)}\right) \text {. }
$$

There is a string of full functors

$$
\text { Chow }^{\text {b }} \rightarrow\left(S_{b}^{-1} \text { Chow }^{\text {eff }}\right)^{\natural} \rightarrow \text { Chow }^{\text {o }}
$$

which become equivalences of categories after inverting the exponential characteristic $p$ of $F$ [29, Th. 2.4.1].

2.3. A full embedding. We now draw other consequences from Theorem 2.1.2. For the reader's convenience, we include a proof of the following generalisation of Lemma 2.1.1, which is in [53, proof of Prop. 2.1.4]:

2.3.1. Theorem. Let $X, Y$ be two smooth projective F-varieties. Then (2.1) is an isomorphism.

Proof. Let $L(Y)$ and $L^{c}(Y)$ be the presheaves with transfers defined in $[53, \S 4.1]$. Then the cokernel of $i_{0}^{*}-i_{1}^{*}$ is clearly isomorphic to $\left.h_{0}(L(Y))(X)\right)$. On the other hand, since $Y$ is projective, the morphism of presheaves $L(Y) \rightarrow L^{c}(Y)$ is an isomorphism. The latter presheaf is canonically isomorphic to $z_{\text {equi }}(Y, 0)$ (compare $\left.[53, \S 4.2]\right)$. The group $C H^{\operatorname{dim} Y}(X \times Y)$, in its turn, is canonically isomorphic to $h_{0}\left(z_{\text {equi }}(X \times\right.$ $Y, \operatorname{dim} X))(\operatorname{Spec} F)$. We therefore have to see that the natural map

$$
h_{0}\left(z_{\text {equi }}(Y, 0)\right)(X) \rightarrow h_{0}\left(z_{\text {equi }}(X \times Y, \operatorname{dim} X)\right)(\operatorname{Spec} F)
$$

is an isomorphism. But the left hand side may be further rewritten

$$
h_{0}\left(z_{\text {equi }}(Y, 0)\right)(X)=h_{0}\left(z_{\text {equi }}(X, Y, 0)\right)(\operatorname{Spec} F)
$$

(cf. [13, bottom p. 142]). The result now follows from [13, Th. 7.1].

2.3.2. Remark. Under resolution of singularities, Theorem 2.3.1 remains true if $X$ is only smooth quasiprojective by replacing [13, Th. 7.1] by [13, Th. 7.4] in the above proof. We shall not need this more refined result.

Consider the full subcategory $\mathbf{S m}^{\text {proj }}$ Cor $\subset$ SmCor whose objects are smooth projective varieties, and its associated homotopy category $\mathcal{H}\left(\mathbf{S m}^{\text {proj }} \mathbf{C o r}\right) \subset \mathcal{H}(\mathbf{S m C o r})$. Then Theorem 2.3.1 yields an isomorphism of categories

$$
\mathcal{H}\left(\mathbf{S m}^{\text {proj }} \text { Cor }\right) \stackrel{\sim}{\longrightarrow} \text { Cor }_{\text {rat }}
$$

where Cor $_{\text {rat }}$ is the category of Chow correspondences (see $\S 2.2$ ). 
2.3.3. Proposition. Let $\mathbf{C o r}_{\mathrm{rat}}^{\mathrm{o}}$ be the category of birational Chow correspondences (see §2.2). Then the identity map on objects extends to a full embedding

$$
\text { Cor }_{\text {rat }}^{\circ} \stackrel{D}{\longrightarrow} \mathrm{BFC}
$$

which fits in the commutative diagram

$$
\begin{array}{ccc}
S_{b}^{-1} \mathcal{H}\left(\mathbf{S m}^{\text {proj }} \text { Cor }\right) & \stackrel{A}{\longrightarrow} & \text { BFC } \\
C \downarrow \text { ? } & & D \uparrow \\
S_{b}^{-1} \mathbf{C o r}_{\text {rat }} & \stackrel{B}{\longrightarrow} \text { Cor }_{\text {rat }}^{\text {o }} .
\end{array}
$$

Here $A$ is the obvious functor, $C$ is induced by (2.3) and $B$ is the functor from [29, Prop. 2.3.7 c)].

Proof. Indeed, in view of Corollary 2.1.3 and [29, Lemma 2.3.6], the isomorphism of Theorem 2.1.2 yields an isomorphism between Hom groups of the two categories Cor $_{\text {rat }}^{\circ}$ and BFC. The proof of Theorem 2.1.2 also shows that this isomorphism defines a (fully faithful) functor, and that (2.4) commutes if we remove $S_{b}^{-1}$ from the left vertical. Therefore, (2.4) commutes.

From Propositions 1.2.1 a) and 2.3.3, we deduce:

2.3.4. Corollary. We have a full embedding

$$
\text { Chow }^{\mathrm{o}} \hookrightarrow \mathrm{HI}^{\mathrm{o}}
$$

which sends the birational motive $h^{\circ}(X)$ of a smooth projective variety $X$ to $h_{0}^{\mathrm{Nis}}(X)$.

\subsection{More equivalences of categories.}

2.4.1. Theorem. In Diagram (2.4), all functors become equivalences of categories after inverting the exponential characteristic $p$ and passing to the pseudo-abelian envelopes.

Thus, after inverting $p$, the categories Chow $^{\mathrm{b}}, S_{b}^{-1}$ Chow $^{\text {eff }}$, Chow $^{\circ}$ and $\mathbf{B F C}^{\natural}$ become equivalent.

Proof. Let $A^{\prime}, B^{\prime}, C^{\prime}, D^{\prime}$ be the corresponding functors. Then $C^{\prime}$ is an isomorphism of categories by (2.3), $B^{\prime}$ is an isomorphism of categories by $\left[29\right.$, Th. 2.4.1] and $D^{\prime}$ is fully faithful by Proposition 2.3.3. To conclude, it remains to show that $D^{\prime}$ is essentially surjective.

Let $X \in \mathbf{S m}$, and let $\bar{X}_{0}$ be a compactification of $X$. For each prime number $l \neq p$, choose by [15] an alteration $\bar{X}_{l} \rightarrow \bar{X}_{0}$ of generic degree prime to $l$, with $\bar{X}_{l}$ smooth. Choose a finite number of primes $l_{1}, \ldots, l_{r}$ such that the gcd of the corresponding degrees $d_{i}$ is a power of $p$, say 
$p^{s}$. Choose a dense open subset $U \subseteq X$ such that $p_{i}: \bar{X}_{l_{i}} \rightarrow \bar{X}_{0}$ is finite over $U$ for all $i$. Let $U_{i}=p_{i}^{-1}(U) \subseteq \bar{X}_{l_{i}}$ and let $V=\coprod_{i=1}^{s} U_{i}$.

Let $\gamma_{i} \in c\left(U_{i}, U\right)$ be the graph of $p_{i \mid U_{i}}$, so that its transpose ${ }^{t} \gamma_{i}$ is still a finite correspondence. Choose integers $n_{i}$ such that $\sum n_{i} d_{i}=p^{s}$. As $\gamma_{i} \circ{ }^{t} \gamma_{i}=d_{i} 1_{U}$, we have

$$
\sum n_{i} \gamma_{i} \circ{ }^{t} \gamma_{i}=p^{s} 1_{U}
$$

Thus, if $a=\bigoplus \gamma_{i} \in c(V, U)[1 / p]$ and $b=\frac{1}{p^{s}} \bigoplus n_{i}{ }^{t} \gamma_{i} \in c(U, V)[1 / p]$, then $b a$ is a projector on $V$ in $\operatorname{SmCor}[1 / p]$, with image isomorphic to $U$.

Let $\bar{X}=\coprod \bar{X}_{l_{i}} \in$ SmCor. The inclusion $V \rightarrow \bar{X}$ becomes an isomorphism in BFC $[1 / p]$, hence the projector $b a$ yields a projector $\pi \in$ End $(\bar{X})$ in the latter category, with image isomorphic to $U$, hence to $X$. Since $D$ is fully faithful, $\pi$ lifts to a projector in $\operatorname{Cor}_{\text {rat }}^{\circ}(\bar{X}, \bar{X})[1 / p]$, thereby concluding the proof.

2.4.2. Corollary. The graph functor $\mathbf{S m} \rightarrow \mathbf{S m C o r}$ induces a functor

$$
S_{b}^{-1} \mathbf{S m}=S_{r}^{-1} \mathbf{S m} \rightarrow \text { Chow }^{\mathrm{o}}[1 / p] .
$$

(See [28, Th. 1.7.2] for the equality $S_{b}^{-1} \mathbf{S m}=S_{r}^{-1} \mathbf{S m}$.)

2.4.3. Remark. In [26, Prop. 4.1], we also proved that the functor

$$
S_{b}^{-1} \mathbf{S m}^{\text {proj }} \text { Cor } \rightarrow S_{b}^{-1} \mathbf{S m C o r}=\mathbf{B F C}
$$

is an equivalence of categories if char $F=0$. Extending this result to positive characteristic (after inverting $p$ and adjoining idempotents) has defied all our attempts, even with the help of [27, Th. 5.1.4]. Fortunately we don't need such a result here, and leave it as a challenge for the interested readers.

\section{Triangulated Birational motives}

In this section, we construct a triangulated category of birational geometric motives $\mathbf{D M}_{\mathrm{gm}}^{\mathrm{o}}$ that we compare with Voevodsky's category $\mathbf{D M}_{\mathrm{gm}}^{\text {eff }}$ of [53]. We also construct a full embedding $\mathbf{B F C} \hookrightarrow \mathbf{D M}_{\mathrm{gm}}^{\mathrm{o}}$; this turns out to be much more elementary than Voevodsky's theory of (effective) triangulated motives, the main reason being that a birational presheaf is automatically a Nisnevich sheaf (Lemma 1.3.2 a)). We organise the exposition in order to highlight this.

In contrast to Voevodsky's approach but like Beilinson-Vologodsky [6], we use unbounded derived categories in order to take advantage of Neeman's yoga of compactly generated triangulated categories [41, 42], which generalises results of Thomason-Trobaugh and Yao. We refer to $\S$ A.1 for general notation and terminology on the latter. 
3.1. Review of effective triangulated motives. We start by recalling Voevodsky's construction of the category $\mathbf{D M}_{\mathrm{gm}}^{\mathrm{eff}}$. In [53], $\mathbf{D M}_{\mathrm{gm}}^{\mathrm{eff}}$ is defined as the pseudo-abelian envelope of the Verdier quotient of $K^{b}$ (SmCor) by the thick subcategory generated by the complexes of the form

$R_{h}:\left[\mathbf{A}_{X}^{1}\right] \stackrel{[p]}{\longrightarrow}[X], X$ smooth;

$R_{M V}:[U \cap V] \rightarrow[U] \oplus[V] \rightarrow[X]$, where $X$ is smooth and $U, V$ are two open subsets such that $X=U \cup V$.

By Balmer-Schlichting [2], $\mathbf{D M}_{\mathrm{gm}}^{\mathrm{eff}}$ is triangulated. The obvious tensor structure on $K^{b}$ (SmCor) induces a tensor structure on $\mathbf{D} \mathbf{M}_{\mathrm{gm}}^{\mathrm{eff}}$.

The canonical embedding of $\mathbf{S m C o r}$ into $K^{b}$ (SmCor) sends SmCor to $\mathbf{D M}_{\mathrm{gm}}^{\mathrm{eff}}$; the image of $[X]$ under this functor is denoted by $M_{\mathrm{gm}}(X)$, or simply here by $M(X)$. The relations $R_{h}$ and the isomorphism of categories (2.3) yield after pseudo-abelianisation a functor [53, Prop. 2.1.4]

$$
\text { Chow }^{\text {eff }} \rightarrow \text { DM }_{\text {gm }}^{\text {eff }}
$$

which sends the Chow motive $h(X)$ of a smooth projective variety $X$ to $M(X)$.

To go further, Voevodsky constructs when $F$ is perfect a full embedding of $\mathbf{D} \mathbf{M}_{\mathrm{gm}}^{\text {eff }}$ into a larger triangulated category $\mathbf{D} \mathbf{M}_{-}^{\text {eff }}$ of sheaftheoretic nature; this allows him to compute Hom groups of $\mathbf{D} \mathbf{M}_{\mathrm{gm}}^{\mathrm{eff}}$ in terms of Nisnevich (or even Zariski) hypercohomology of certain complexes. As a byproduct, the functor (3.1) is fully faithful. We recall part of this story in $\S 3.4$.

We shall now see that the perfectness of $F$ is not needed for the corresponding properties of triangulated birational motives.

3.2. Triangulated birational motives. Let us apply Proposition A.4.1 with $\mathcal{A}=$ SmCor. In this case, $\operatorname{Mod}-\mathcal{A}=\mathrm{PST}$, the category of presheaves with transfers. Thus we get that the Yoneda functor

$$
K^{b}(\text { SmCor }) \rightarrow D(\text { PST })
$$

is fully faithful and has dense image, whose pseudo-abelian envelope consists precisely of the compact objects of the right hand side.

3.2.1. Definition. Let $R_{\mathrm{o}} \subset K^{b}$ (SmCor) be the class of complexes $[U] \stackrel{j}{\longrightarrow}[X]$, where $j$ is an open immersion with dense image. We denote by ( $c f$. Definition A.1.2 for the notation $\left.\left\langle R_{\mathrm{o}}\right\rangle,\left\langle L\left(R_{\mathrm{o}}\right)\right\rangle^{\oplus}\right)$ :

- $\mathbf{D M}_{\mathrm{gm}}^{\mathrm{o}}$ the pseudo-abelian envelope of the Verdier quotient of $K^{b}(\mathbf{S m C o r})$ by $\left\langle R_{\mathrm{o}}\right\rangle$. We denote the image of $[X]$ in $\mathbf{D M}_{\mathrm{gm}}^{\mathrm{o}}$ by $M^{\circ}(X)$. (It is triangulated by [2].) 
- $\mathbf{D M}^{\circ}$ the localisation of $D(\mathbf{P S T})$ with respect to $\left\langle L\left(R_{\mathrm{o}}\right)\right\rangle^{\oplus}$. We define $\mathbf{D} \mathbf{M}_{-}^{\mathrm{o}}$ similarly, using $D^{-}(\mathbf{P S T})$ instead of $D(\mathbf{P S T})$.

For the next theorem, recall the notation

$$
D_{\mathcal{B}}(\mathcal{A})=\left\{C \in D(\mathcal{A}) \mid H^{i}(C) \in \mathcal{B} \quad \forall i \in \mathbf{Z}\right\}
$$

if $\mathcal{B}$ is a strictly full subcategory of an abelian category $\mathcal{A}$ : this is a triangulated subcategory of $D(\mathcal{A})$ provided $\mathcal{B}$ is thick in $\mathcal{A}$, i.e. given a short exact sequence in $\mathcal{A}$, if two terms belong to $\mathcal{B}$ then so does the third. Note that $\mathbf{H I}^{\mathrm{o}}$ is thick in PST.

3.2.2. Theorem. Let $\gamma: \mathbf{S m C o r} \rightarrow \mathbf{B F C}$ be the localisation functor. a) The functor $K^{b}(\gamma)^{\natural}: K^{b}(\mathbf{S m C o r})^{\natural} \rightarrow K^{b}(\mathbf{B F C})^{\natural}$ factors through $\mathbf{D M}_{\mathrm{gm}}^{\mathrm{o}}$. The total derived functor $L \gamma_{!}: D(\mathbf{P S T}) \rightarrow D\left(\mathbf{H I}^{\circ}\right)$ factors through $\mathbf{D M}^{\mathrm{o}}$. This yields a naturally commutative diagram

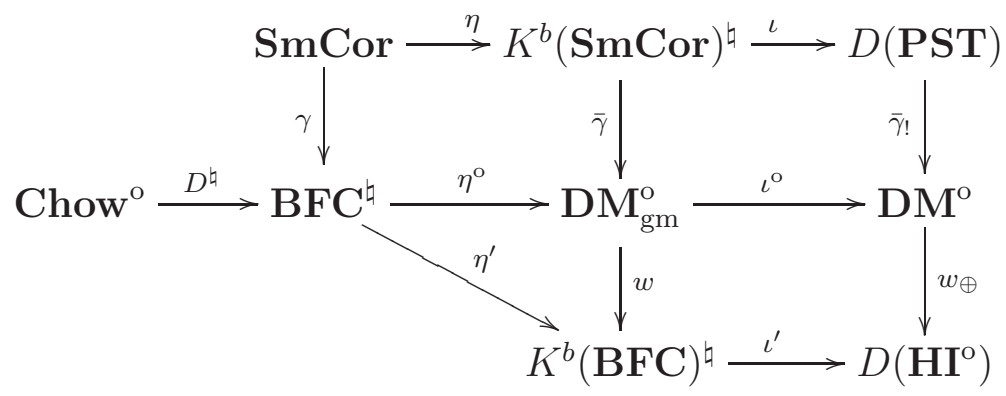

in which all functors not starting from Chow $^{\circ}$, SmCor or $\mathbf{B F C} \mathbf{C}^{\natural}$ are triangulated.

b) The functors $\eta, \eta^{\circ}$ and $\eta^{\prime}$ are fully faithful. The functors $\iota, \iota^{\circ}$ and $\iota^{\prime}$ are fully faithful with dense images, and identify their domains with the full subcategory of compact objects of their range.

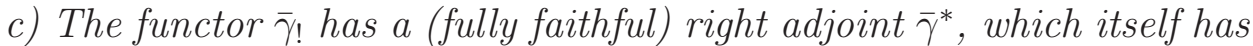
a right adjoint $\bar{\gamma}_{*}$. The essential image of $\bar{\gamma}^{*}$ is $D_{\mathbf{H I}^{\circ}}(\mathbf{P S T})$, where $\mathbf{H I}^{\mathrm{o}}$ is embedded in PST by means of $\gamma^{*}$.

d) Via $\bar{\gamma}^{*}$, the natural $t$-structure of D(PST) induces a t-structure on $\mathbf{D M}^{\mathrm{o}}$, with heart $\mathbf{H I}^{\mathrm{o}}$; the functor $\bar{\gamma}_{!}$(resp. $\left.\bar{\gamma}_{*}\right)$ is right (resp. left) t-exact.

e) For $X, Y \in \mathbf{S m}$ and $\mathcal{F} \in \mathbf{H I}^{\circ}$, we have:

$$
\begin{aligned}
& \operatorname{DM}^{\mathrm{o}}\left(M^{\mathrm{o}}(X), \mathcal{F}[q]\right)= \begin{cases}0 & \text { for } q \neq 0 \\
\mathcal{F}(X) & \text { for } q=0,\end{cases} \\
& \operatorname{DM}^{\mathrm{o}}\left(M^{\mathrm{o}}(X), M^{\mathrm{o}}(Y)[i]\right)=0 \quad \text { for } i>0 \\
& \mathcal{H}^{i}\left(\bar{\gamma}^{*} M^{\mathrm{o}}(Y)\right)=0 \quad \text { for } i>0 .
\end{aligned}
$$


f) If $Y$ is proper, we have

$$
\begin{aligned}
\operatorname{DM}^{\mathrm{o}}\left(M^{\mathrm{o}}(X), M^{\mathrm{o}}(Y)\right) & =C H_{0}\left(Y_{F(X)}\right) \\
\mathcal{H}^{0}\left(\bar{\gamma}^{*} M^{\mathrm{o}}(Y)\right) & =h_{0}^{\mathrm{Nis}}(Y) .
\end{aligned}
$$

The functor $D^{\natural}$ is fully faithful (hence so is $\eta^{\circ} D^{\natural}$ ).

g) The obvious functor

$$
\varphi: D\left(\mathbf{H I}^{\mathrm{o}}\right) \rightarrow D_{\mathbf{H I}^{\circ}}(\mathbf{P S T}) \simeq \mathbf{D M}^{\mathrm{o}}
$$

is right adjoint to $w_{\oplus}$, t-exact and conservative; it induces the identity on the hearts.

h) The functor $w_{\oplus}$ is right t-exact and induces the identity on the hearts; its restriction to $\mathbf{D M}_{-}^{\circ}$ is conservative. The functor $w$ is conservative as well.

Proof. Everything follows directly from Proposition A.4.1 and Theorem A.4.2, except for $\mathrm{f}$ ) which follows from Theorem 2.1.2, Corollary 2.1.3 and Proposition 2.3.3.

3.2.3. Remark. By theorem 2.4.1, $D^{\natural}$ becomes essentially surjective after inverting the exponential characteristic $p$.

To Theorem 3.2.2, we add:

3.2.4. Proposition. The $\otimes$-structure on $\mathbf{S m C o r}$ induces a $\otimes$-structure on all categories in the diagram of Theorem 3.2.2, and all functors in this diagram are $\otimes$-functors. The $\otimes$-structures are compatible with the triangulated structures when applicable.

Proof. Indeed, if $U \stackrel{j}{\longrightarrow} X$ is a dense open immersion, then $U \times Y \stackrel{j \times 1_{Y}}{\longrightarrow}$ $X \times Y$ is also a dense open immersion for any $Y \in \mathbf{S m}$.

The next result is deeper:

3.2.5. Proposition. The thick subcategory $\left\langle R^{\circ}\right\rangle^{\natural} \subset \mathbf{D M}_{\mathrm{gm}}^{\mathrm{eff}}$ contains all motives of the form $M(1):=M \otimes \mathbf{Z}(1)$. If $F$ is perfect, this is an equality and the functor $\mathbf{D M}_{\mathrm{gm}}^{\mathrm{eff}} \rightarrow\left\langle R^{\mathrm{o}}\right\rangle^{\natural}$ given by $M \mapsto M(1)$ is an equivalence of categories. Similarly, $\mathbf{D M}^{\mathrm{eff}}(1) \subseteq\left\langle R^{\mathrm{o}}\right\rangle^{\oplus}$ with equality when $F$ is perfect.

Proof. By density, the case of $\left\langle R^{\circ}\right\rangle^{\oplus}$ reduces to that of $\left\langle R^{\circ}\right\rangle^{\natural}$. Let $\mathcal{D}$ be the full subcategory of $\mathbf{D M}_{\mathrm{gm}}^{\mathrm{eff}}$ consisting of the motives of the form $M(1)$. Since $\mathbf{Z} \oplus \mathbf{Z}(1)[2]=M^{\mathrm{o}}\left(\mathbf{P}^{1}\right)=M^{\mathrm{o}}\left(\mathbf{A}^{1}\right)=\mathbf{Z}$ in $\mathbf{D M}_{\mathrm{gm}}^{\mathrm{o}}, \mathbf{Z}(1)=0$ in $\mathbf{D M}_{\mathrm{gm}}^{\mathrm{o}}$. Therefore $\mathcal{D} \subseteq\left\langle R^{\mathrm{o}}\right\rangle^{\natural}$ by Proposition 3.2.4.

To see the converse inclusion when $F$ is perfect, we first prove that $\mathcal{D}$ is a triangulated subcategory of $\mathbf{D M}_{\mathrm{gm}}^{\mathrm{eff}}$. We have to show that, if 
$M, N \in \mathbf{D M}_{\mathrm{gm}}^{\mathrm{eff}}$ and $f \in \operatorname{Hom}(M(1), N(1))$, then the cone of $f$ is of the form $P(1)$. This follows from the cancellation theorem of [55]. The cancellation theorem also shows that $M \mapsto M(1)$ yields an equivalence of categories $\mathbf{D M}_{\mathrm{gm}}^{\mathrm{eff}} \stackrel{\sim}{\longrightarrow} \mathcal{D}$.

We now have to prove that $M(U) \stackrel{j_{*}}{\longrightarrow} M(X)$ is an isomorphism in $\mathbf{D M}_{\mathrm{gm}}^{\mathrm{eff}} / \mathcal{D}$ for any open immersion $j$. We argue by Noetherian induction on the (reduced) closed complement $Z$ in a standard way. For simplicity, let us say that the open immersion $j$ is pure if $Z$ is smooth. If $j$ is pure, then the cone of $j_{*}$ is isomorphic to $M(Z)(c)[2 c]$, where

$c=\operatorname{codim}_{X} Z$ by the Gysin exact triangle of [53, Prop. 3.5.4], so the claim is true in this case. In general, filtering $Z$ by its successive singular loci, we may write $j$ as a composition of pure open immersions, and the claim follows.

\subsection{Relationship between effective and birational triangulated}

motives. We already introduced three classes $R_{h}, R_{M V}$ and $R_{\mathrm{O}}$ of objects of $K^{b}$ (SmCor). Here we shall use two others:

- $R_{\mathrm{Nis}}=\{[B] \rightarrow[A] \oplus[Y] \rightarrow[X]\}$, where

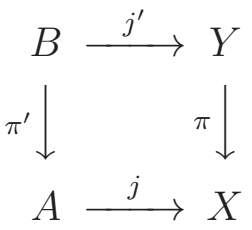

is an upper-distinguished square in the sense of [36, Def. 12.5] (also called elementary distinguished square in [40, p. 96, Def. $1.3])$.

- $R=R_{h} \cup R_{\mathrm{Nis}}$.

3.3.1. Lemma. We have the following inclusions:

(i) $R_{M V} \subset R_{\mathrm{Nis}}$;

(ii) $\left\langle R_{\mathrm{Nis}}\right\rangle \subset\left\langle R_{\mathrm{o}}\right\rangle$;

(iii) $\left\langle R_{h}\right\rangle \subset\left\langle R_{\mathrm{o}}\right\rangle$;

(iv) $\langle R\rangle \subset\left\langle R_{\mathrm{o}}\right\rangle$.

Moreover, the classes $R_{h}, R_{M V}, R_{\mathrm{Nis}}, R$ and $R_{\mathrm{o}}$ are stable under $-\otimes[X]$ for any $X \in \mathbf{S m}$.

Proof. This is essentially a reformulation of Lemmas 1.3.2 and 1.3.3. (i) is obvious. (ii) follows from the fact that, in (3.4), $j$ and $j^{\prime}$ are open immersions. (iii) follows from [28, Th. 1.7.2]. (iv) follows from (ii) and (iii). The last statement is obvious (and already observed for $R_{\mathrm{o}}$ ).

We also recall the following important fact [6, p. 1749, (4.3.1)]: 
3.3.2. Proposition. Let La $: D(\mathbf{P S T}) \rightarrow D(\mathbf{N S T})$ be the functor induced by the exact functor a of (1.5). Then La is a localisation, with kernel $\left\langle R_{\mathrm{Nis}}\right\rangle^{\oplus}$.

It is now convenient for the exposition to introduce the "Nisnevich competitor" of $\mathbf{D M}_{\mathrm{gm}}^{\mathrm{eff}}$ :

3.3.3. Definition. The category $\left(\mathbf{D M}_{\mathrm{gm}}^{\mathrm{eff}}\right)_{\mathrm{Nis}}$ is the pseudo-abelian envelope of the Verdier quotient $K^{b}$ (SmCor) $) /\langle R\rangle$. We also define $\mathbf{D M}^{\text {eff }}=$ $D(\mathbf{P S T}) /\langle L(R)\rangle^{\oplus}$ and $\mathbf{D M}_{-}^{\mathrm{eff}}=D^{-}(\mathbf{P S T}) /\langle L(R)\rangle^{\oplus}$.

From Proposition 3.3.2, we deduce:

3.3.4. Proposition. The categories $\mathbf{D} \mathbf{M}^{\mathrm{eff}}$ and $\mathbf{D} \mathbf{M}_{-}^{\mathrm{e} f \mathrm{f}}$ are respectively equivalent to $D(\mathbf{N S T}) /\left\langle L\left(R_{h}\right)\right\rangle^{\oplus}$ and $D^{-}(\mathbf{N S T}) /\left\langle L\left(R_{h}\right)\right\rangle^{\oplus}$. In particular, $\mathbf{D M}_{-}^{\mathrm{eff}}$ coincides with the category defined in [36, Def. 14.1].

Proposition 3.3.2 and Lemma 3.3.1 yield the following naturally commutative diagram, which summarises what we got so far (the notations $L C$ and $\nu_{\leq 0}$ will be explained in $\left.\S 3.6^{4}\right)$ :

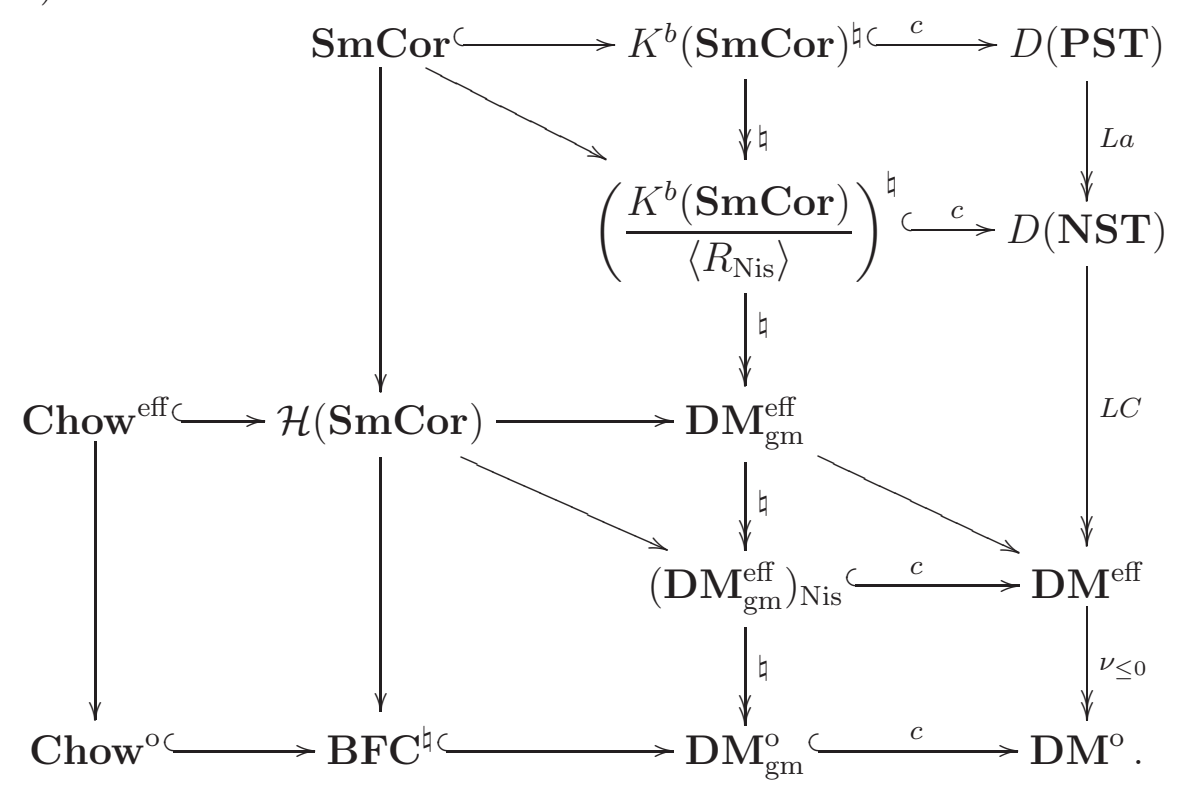

In (3.5), all categories are $\otimes$ categories and all functors are $\otimes$ functors. In the two right columns, the categories and functors are triangulated. We use $\longleftrightarrow$ (resp. $\longrightarrow, \stackrel{\natural}{\longrightarrow)}$ ) to denote a full embedding

\footnotetext{
${ }^{4}$ In [53, Prop. 3.2.3], the bounded above version of the first functor is denoted by $\mathbf{R} C$, but we prefer the notation $L C$ as it is a left adjoint.
} 
(resp. a localisation, a localisation followed by taking pseudo-abelian envelope), and the letter $c$ means that the corresponding functor is a dense embedding of the full subcategory of compact objects (see $\S \mathrm{A} .1$ ).

The full and dense embeddings on the top and bottom rows come from Theorem 3.2.2, while the two other full and dense embeddings follow from applying Theorem A.2.2.

We also have:

3.3.5. Theorem. The functors $L a, L C$ and $\nu_{\leq 0}$ of Diagram 3.5 have (fully faithful) right adjoints $R k, i$ and $i^{\circ}$, which in turn have right adjoints.

Proof. For $L a$ and the compositions $L C L a, \nu_{\leq 0} L C L a$, this follows from the dense embedding (3.2) and Theorem A.2.6. For the individual functors, we now get the adjoints from Proposition 3.3.6 below.

\subsubsection{Proposition. Let}

$$
\mathcal{C} \stackrel{F}{\longrightarrow} \mathcal{D} \stackrel{G}{\longrightarrow} \mathcal{E}
$$

be a sequence of categories and functors; let $H=G F$.

a) Suppose that $H$ has a right adjoint $H_{*}$ and that $G$ is fully faithful. Then $F$ has a right adjoint, given by $F_{*}=H_{*} G$.

b) Suppose that $H$ and $F$ have right adjoints $H^{*}$ and $F^{*}$ and that $F$ is a localisation. Then $G$ has a right adjoint, given by $G^{*}=F H^{*}$.

Proof. a) For $c \in \mathcal{C}$ and $d \in \mathcal{D}$, we have a map

$$
\mathcal{D}(F c, d) \stackrel{G}{\longrightarrow} \mathcal{E}(G F c, G d) \stackrel{\sim}{\longrightarrow} \mathcal{C}\left(c, H_{*} G d\right)
$$

which is natural in $d$ and $c$, and $G: \mathcal{D}(F c, d) \rightarrow \mathcal{E}(G F c, G d)$ is bijective since $G$ is fully faithful.

b) The argument is similar but a little more delicate: let $d \in \mathcal{D}$ and $e \in \mathcal{E}$. Since $F$ is a localisation, it is surjective so that $d=F c$ for some $c \in \mathcal{C}$. We then have a map

$$
\mathcal{E}(G d, e)=\mathcal{E}(G F c, e) \stackrel{\sim}{\longrightarrow} \mathcal{C}\left(c, H^{*} e\right) \stackrel{F}{\longrightarrow} \mathcal{D}\left(F c, F H^{*} e\right)=\mathcal{D}\left(d, F H^{*} e\right) .
$$

By adjunction, the map $F: \mathcal{C}\left(c, H^{*} e\right) \rightarrow \mathcal{D}\left(F c, F H^{*} e\right)$ is converted into the map $\mathcal{C}\left(c, H^{*} e\right) \rightarrow \mathcal{C}\left(c, F^{*} F H^{*} e\right)$ induced by the unit morphism $H^{*} e \rightarrow F^{*} F H^{*} e$. Let us show that the latter is an isomorphism. Since $F$ is a localisation, $F^{*}$ is fully faithful $[16$, I.1.4] and it suffices to see that $H^{*} e \in \operatorname{Im} F^{*}$, which is true since

$$
\operatorname{Im} F^{*}=\{\gamma \in \mathcal{C} \mid \mathcal{C}(s, \gamma) \text { is bijective for all } s
$$

$$
\text { such that } F(s) \text { is invertible }\} \supseteq \operatorname{Im} H^{*} \text {. }
$$


3.3.7. Remarks. 1) Passing to the categories of presheaves, one can see that the existence of $H^{*}$ is not necessary in the hypothesis of Proposition 3.3.6 b).

2) Using standard arguments for unbounded triangulated categories [47], one sees that the right adjoint $R k$ of $L a$ is the total derived functor of $k:$ NST $\hookrightarrow$ PST.

\subsection{The case of a perfect field. We have:}

3.4.1. Theorem. Suppose F perfect. Then, in Diagram (3.5):

(1) The composite functor $\mathbf{D} \mathbf{M}_{\mathrm{gm}}^{\mathrm{eff}} \rightarrow\left(\mathbf{D M}_{\mathrm{gm}}^{\mathrm{eff}}\right)_{\mathrm{Nis}} \rightarrow \mathbf{D M}^{\mathrm{eff}}$ is fully faithful.

(2) The functor $\mathbf{D} \mathbf{M}_{\mathrm{gm}}^{\mathrm{eff}} \rightarrow\left(\mathbf{D M}_{\mathrm{gm}}^{\mathrm{eff}}\right)_{\mathrm{Nis}}$ is an equivalence of categories.

(3) The functor Chow $^{\text {eff }} \rightarrow \mathrm{DM}_{\mathrm{gm}}^{\mathrm{eff}}$ is fully faithful.

All this is summarised in the following simpler diagram:

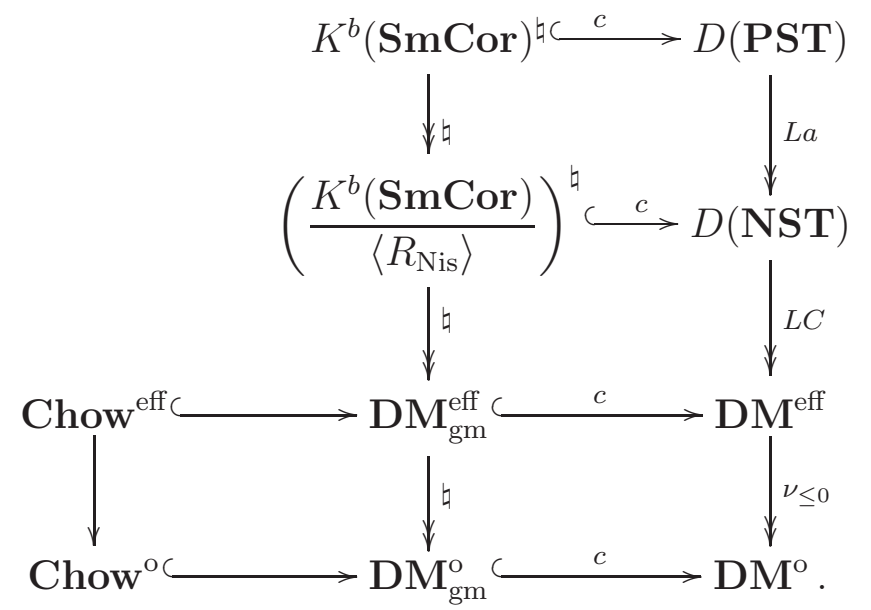

Finally, the canonicalt-structure of D(NST) induces a t-structure with heart $\mathbf{H I}$ on $\mathbf{D M}^{\text {eff }}$ via the right adjoint $i$ to $L C$; the latter induces on $\mathrm{DM}^{\mathrm{O}}$ the -structure of Theorem 3.2.2 $d$ ) via the right adjoint $i^{\mathrm{O}}$ to $\nu_{\leq 0}$.

Proof. This summarises some of the main results of Voevodsky. Namely, (1) is [53, Th. 3.2.6 1], and (2) follows from (1) since the first (resp. second) functor in (1) is a localisation (resp. is fully faithful) by Diagram (3.5). (3) is proven in [53, Cor. 4.2.6] and [36, Prop. 20.1] under resolution of singularities; in [6, Cor. 6.7.3], this is extended to any perfect 
field by a simple duality argument. As for $t$-structures, the first statement is [53, comment after Prop. 3.1.13 $]^{5}$. The second one amounts to say that $i^{\mathrm{o}}$ is $t$-exact. This follows from Theorem $3.2 .2 \mathrm{~d}$ ), namely the $t$-exactness of $\bar{\gamma}^{*}=R k i i^{\circ}$, and from the exactness of the sheafification functor $a: \mathbf{P S T} \rightarrow \mathbf{N S T}$. Namely, let $C \in\left(\mathbf{D M}^{\mathrm{o}}\right)^{\leq 0}$. By Theorem 3.2.2 d), $\mathcal{H}^{i}\left(R k i i^{\circ} C\right)=0$ for $i>0$, hence $\mathcal{H}^{i}\left(i i^{\circ} C\right)=a \mathcal{H}^{i}\left(R k i i^{\circ} C\right)=0$ for $i>0$ as well, and $i^{\mathrm{o}} C \in\left(\mathbf{D M}^{\text {eff }}\right) \leq 0$. The reasoning is the same to get $i^{\mathrm{o}}\left(\mathbf{D M}^{\mathrm{o}}\right)^{\geq 0} \subset\left(\mathbf{D M}^{\text {eff }}\right) \geq 0$.

3.4.2. Remarks. 1) Consider the $t$-structures of Theorem 3.4.1. The right adjoint $i i^{\circ}$ of $\nu_{\leq 0} L C$ is $t$-exact. On the other hand, $a: D(\mathbf{P S T}) \rightarrow$ $D$ (NST) is $t$-exact but its right adjoint $R k$ is clearly not $t$-exact. Neither is the composition $R k \circ i$ when $F$ is perfect: for example, $\mathcal{H}^{1}\left(R k \circ i\left(\mathbb{G}_{m}\right)\right)$ is the presheaf $X \mapsto \operatorname{Pic}(X)$. However, the composition of all right adjoints $R k \circ i \circ i^{\circ}$ is $t$-exact, as just used in the above proof.

2) In [29, Th. 4.3.3], we showed that the functor Chow ${ }^{\text {eff }} \rightarrow$ Chow $^{\text {o }}$ does not have a right adjoint, even after tensoring Hom groups with Q; more precisely, this right adjoint is not defined at the motive of a suitable smooth projective 3 -fold. We shall strengthen this result in Remark 3.6.5 by showing that the right adjoint of $\mathbf{D M}_{\mathrm{gm}}^{\mathrm{eff}} \rightarrow \mathbf{D M}_{\mathrm{gm}}^{\mathrm{o}}$ is not defined at the motive of a suitable smooth projective 3-fold, even after tensoring with $\mathrm{Q}$.

3.5. The essential images of $i$ and $i^{\mathrm{o}}$. The following proposition computes some Hom groups in $D(\mathbf{N S T}), \mathbf{D M}^{\text {eff }}$ and $\mathbf{D M}^{\mathbf{0}}$ :

3.5.1. Proposition. Let $X$ be a smooth scheme over $F$ and let $C$ be an object of $D(\mathbf{N S T})$ (resp. $\left.\mathbf{D} \mathbf{M}^{\mathrm{eff}}, \mathbf{D M}^{\mathrm{o}}\right)$. Then there is a canonical isomorphism

$$
\begin{aligned}
D(\mathbf{N S T})(L(X), C) & \simeq H_{\mathrm{Nis}}^{0}(X, C) \\
\left(\text { resp. } \mathbf{D M}^{\mathrm{eff}}(M(X), C)\right. & \simeq H_{\mathrm{Nis}}^{0}(X, C), \\
\mathbf{D M}^{\mathrm{o}}\left(M^{\mathrm{o}}(X), C\right) & \left.\simeq H_{\mathrm{Nis}}^{0}(X, C)\right) .
\end{aligned}
$$

(Note that we write $H_{\mathrm{Nis}}^{i}(X, C)$ for the Nisnevich hypercohomology of $C$, which is sometimes written $\mathbb{H}_{\mathrm{Nis}}^{i}(X, C)$.)

Proof. This is [53, Prop. 3.1.8] when $C$ is bounded above; but the same argument works for an unbounded $C$ by replacing an injective resolution of $C$ by a $K$-injective resolution in the sense of Spaltenstein

\footnotetext{
${ }^{5}$ Recall that all the above relies on the highly nontrivial fact that a homotopy invariant Nisnevich sheaf with transfers is strictly homotopy invariant: [53, Th. 3.1.12 1] or [36, Th. 13.8].
} 
[47, Th. 4.5 and Rk. 4.6], compare [36, Ex. 13.5]. The other statements follow by adjunction.

We shall also need:

3.5.2. Proposition. The internal Hom of D(NST) induces an internal Hom on $\mathbf{D M}^{\mathrm{eff}}$ via $i$. We denote it by $\underline{\mathrm{Hom}}_{\mathrm{eff}}$.

Proof. This follows by adjunction from the fact that $L C: D(\mathbf{N S T}) \rightarrow$ $\mathbf{D M}^{\text {eff }}$ is a $\otimes$-functor, as observed just below (3.5).

3.5.3. Corollary. The essential image of $i: \mathbf{D M}^{\mathrm{eff}} \rightarrow D(\mathbf{N S T})$ (resp. $\left.i^{\mathrm{o}}: \mathbf{D M}^{\mathrm{O}} \rightarrow \mathbf{D M}^{\mathrm{eff}}\right)$ is the full subcategory of those complexes $C$ such that

$$
H_{\mathrm{Nis}}^{*}(X, C) \stackrel{\sim}{\longrightarrow} H_{\mathrm{Nis}}^{*}\left(X \times \mathbf{A}^{1}, C\right)
$$

for all smooth $X$ (resp. such that

$$
H_{\mathrm{Nis}}^{*}(X, C) \stackrel{\sim}{\longrightarrow} H_{\mathrm{Nis}}^{*}(U, C)
$$

for any dense open immersion $U \rightarrow X$ of smooth varieties).

Proof. This follows from Proposition 3.5.1 and Theorem A.2.6 (ii).

Here is an alternate description of $i^{\mathrm{o}} \mathbf{\mathbf { D }} \mathbf{M}^{\mathrm{o}}$. The following lemma follows from Proposition 3.2.5 and Theorem A.2.6:

3.5.4. Lemma. $i^{\mathrm{o}} \mathbf{D M}^{\mathrm{o}} \subseteq\left\{C \in \mathbf{D M}^{\mathrm{eff}} \mid \underline{\operatorname{Hom}}_{\mathrm{eff}}(\mathbf{Z}(1), C)=0\right\}$ (see Proposition 3.5.2). If $F$ is perfect, this inclusion is an equality.

As was already observed in [20] and [24], this implies that the terms of the "associated graded of the slice filtration" on an object of $\mathbf{D} \mathbf{M}^{\text {eff }}$ are twists of birational motives.

3.6. Computing $i^{\mathrm{o}} \nu_{\leq 0}$. In this subsection, we assume $F$ perfect. We first recall Voevodsky's computation of $i L C$ in this case. Recall that, if $\mathcal{F} \in \mathbf{N S T}$, the Suslin complex $\underline{C}_{*}(\mathcal{F})$ of $\mathcal{F}$ is the (chain) complex of Nisnevich sheaves with transfers given in degree $n$ by

$$
C_{n}(\mathcal{F})(X)=\mathcal{F}\left(X \times \Delta^{n}\right)
$$

where the differentials are induced by linear combinations of the face maps [53, p. 207], [36, Def. 2.12]. If $K$ is a bounded below chain complex (= bounded above cochain complex) of Nisnevich sheaves with transfers, we can extend this definition by

$$
\underline{C}_{*}(K)=\operatorname{Tot}\left(p \mapsto \underline{C}_{*}\left(K_{p}\right)\right) .
$$


Finally, if $K \in C(\mathbf{N S T})$, we define

$$
\underline{C}_{*}(K)=\text { hocolim } \operatorname{Tot} \underline{C}_{*}\left(\tau_{\leq n} K\right)
$$

(see [7] for hocolim).

This defines an endofunctor of $D(\mathbf{N S T})$. Then:

3.6.1. Proposition ([53, Prop. 3.2.3]). For any $K \in D(\mathbf{N S T})$, we have a natural isomorphism

$$
i L C(K) \simeq \underline{C}_{*}(K)
$$

We now study the functor $\nu_{\leq 0}$ along with its right adjoint $i^{\mathrm{o}}$. In this case, the story is much simpler.

Consider the inclusion functor $\mathbf{D} M^{\mathrm{eff}}(1) \hookrightarrow \mathbf{D M}^{\mathrm{eff}}$. Using the cancellation theorem, its right adjoint is trivial to write down: it is given by

$$
M \mapsto \underline{\operatorname{Hom}}_{\mathrm{eff}}(\mathbf{Z}(1), M)(1)
$$

where $\underline{\text { Hom }}_{\text {eff }}$ is the internal Hom in $\mathbf{D M}^{\text {eff }}$ (see Proposition 3.5.2). From Proposition 3.2.5 and Theorem A.2.6 (iii), we then immediately get a formula for $i^{\mathrm{o}} \nu_{\leq 0}$ :

3.6.2. Proposition. If $F$ is perfect, we have an exact triangle for any $M \in \mathbf{D M}^{\mathrm{eff}}$

$$
\underline{\operatorname{Hom}}_{\text {eff }}(\mathbf{Z}(1), M)(1) \rightarrow M \rightarrow i^{\mathrm{o}} \nu_{\leq 0} M \stackrel{+1}{\longrightarrow} .
$$

In [20], this appears as part of the description of the slice filtration on $M$.

3.6.3. Remark. In [24, Rk. 2.2.6] there is a different "computation" of the functor $i^{\circ} \nu_{\leq 0}$, in the spirit of Proposition 3.6.1: for a smooth $F$-variety $X$ with function field $K$, write $\hat{\Delta}_{X}^{n}=\hat{\Delta}_{K}^{n}$ for the semilocalisation of $\Delta_{K}^{n}$ at the vertices. This defines a sub-cosimplicial scheme of $\Delta_{K}^{*}$ and $\Delta_{X}^{*}$. Thus, for $\mathcal{F} \in \mathbf{P S T}$, we may define

$$
\hat{C}_{*}(\mathcal{F})(X)=\mathcal{F}\left(\hat{\Delta}_{X}^{*}\right)
$$

the $n$-th term of this chain complex is $\hat{C}_{n}(\mathcal{F})(X)=\mathcal{F}\left(\hat{\Delta}_{X}^{n}\right)$ (defined by an inductive limit), and the differentials are induced by the face maps. We may then extend $\hat{C}_{*}$ to $C(\mathbf{P S T})$ as above. Then, for $N \in C(\mathbf{P S T})$ with homotopy invariant homology presheaves, we have a canonical isomorphism

$$
i^{\mathrm{o}} \nu_{\leq 0} \operatorname{LaN}(X) \simeq \hat{C}_{*}(N) \in D(\mathbf{A b}) .
$$

3.6.4. Remark. Theorem 3.2.2 e) and f) identifies some of the homology (pre)sheaves $h_{q}^{\mathrm{o}}(Y)$ of $M^{\mathrm{o}}(Y)$ for a smooth proper variety $Y$ : they are 0 for $q<0$ and $h_{0}^{\circ}(Y)=h_{0}^{\mathrm{Nis}}(Y)$. One may wonder about $q>0$. If 
$Y$ is a curve, we have $h_{q}^{\mathrm{Nis}}(Y)=0$ for $q>1$ and $h_{1}^{\mathrm{Nis}}(Y)=p_{*} p^{*} \mathbb{G}_{m}$ by [53, Th. 3.4.2], where $p$ is the structural morphism. Thus we get an exact triangle

$$
\left(p_{*} \mathbf{Z}\right)(1)[2] \rightarrow M(Y) \rightarrow h_{0}^{\mathrm{Nis}}(Y)[0] \stackrel{+1}{\longrightarrow}
$$

whence $M^{\mathrm{o}}(Y) \stackrel{\sim}{\longrightarrow} h_{0}^{\mathrm{Nis}}(Y)[0]$ by Corollary 2.1.3. In this case, we therefore find $h_{q}^{\mathrm{o}}(Y)=0$ for all $q \neq 0$.

This is no longer true when $Y$ is a surface. Indeed, at least if $F$ is algebraically closed, one can then produce an isomorphism

$$
h_{1}^{\mathrm{o}}(Y) \simeq H_{\text {ind }}^{3}(Y, \mathbf{Z}(2))
$$

where the right hand side is the quotient of $H^{3}(Y, \mathbf{Z}(2)) \simeq H^{1}\left(Y, \mathcal{K}_{2}\right)$ by the image of $\operatorname{Pic}(Y) \otimes F^{*}$. See [30, Th. 4.1].

3.6.5. Remark. We can now justify Remark 3.4.2 2): let $M \in \mathbf{D M}_{\mathrm{gm}}^{\mathrm{o}}$. By the universal property of a right adjoint and the full faithfulness of $\mathbf{D M}_{\mathrm{gm}}^{\mathrm{o}} \rightarrow \mathbf{D M}^{\mathrm{o}}$, the right adjoint of $\mathbf{D M}_{\mathrm{gm}}^{\mathrm{eff}} \rightarrow \mathbf{D M}_{\mathrm{gm}}^{\mathrm{o}}$ is defined at $M$ if and only if $i^{\mathrm{o}} \nu_{\leq 0} M \in \mathrm{DM}_{\mathrm{gm}}^{\mathrm{eff}}$ (and then $i^{\mathrm{o}} \nu_{\leq 0} M$ is the value of this right adjoint). Suppose $F$ perfect. By the exact triangle of Proposition 3.6.2, the latter is equivalent to $\underline{\operatorname{Hom}}_{\mathrm{eff}}(\mathbf{Z}(1), M)(1) \in \mathbf{D M}_{\mathrm{gm}}^{\mathrm{eff}}$.

Let us show that this fails for $M=M^{\circ}(X), X$ a suitable 3-dimensional smooth projective variety. ${ }^{6}$ In $[19$, App. A], Ayoub proved that if the Griffiths group of $X$ is not finitely generated, then $\underline{\operatorname{Hom}}_{\mathrm{eff}}(\mathbf{Z}(1), M(X))$ is not compact in $\mathbf{D} \mathbf{M}^{\text {eff }}$, hence is not in $\mathbf{D M}_{\text {gm }}^{\text {eff }}$; this works even with Q-coefficients. (There are several examples of such $X$, starting from a general quintic hypersurface in $\mathbf{P}^{4}$ over $F=\mathbf{C}$ which is the original example of Clemens and Griffiths.) It suffices to show that the same then holds for $\underline{\operatorname{Hom}}_{\mathrm{eff}}(\mathbf{Z}(1), M)(1)$ : but this is clear by the cancellation theorem [55], since $K \mapsto K(1)$ commutes with infinite direct sums.

3.7. $t$-structures and projective objects. (Compare $[6$, p. 1737, Footnote 17].)

Let $\mathcal{S}$ be a triangulated category with a $t$-structure, with heart $\mathcal{A}$. Let us say that an object $S \in \mathcal{S}$ is projective (with respect to the $t$ structure) if $\mathcal{S}(S, A[i])=0$ for all $A \in \mathcal{A}$ and $i \neq 0$. Theorem 3.2.2 e), and more generally Theorem A.4.2 e), gives such examples. If $S$ is bounded above (i.e. $S \in \mathcal{S}^{\leq n}$ for some $n$ ), an inductive Yoneda-style argument shows that $S \in \mathcal{S}^{\leq 0}$. Moreover, for any $A \in \mathcal{A}$, one has

$$
\mathcal{S}\left(H^{0}(S), A\right) \stackrel{\sim}{\longrightarrow} \mathcal{S}(S, A), \quad \mathcal{S}\left(H^{0}(S), A[1]\right)=0
$$

\footnotetext{
${ }^{6}$ Recall that the right adjoint is defined at $M^{\mathrm{o}}(X)$ for $X$ smooth projective of dimension $\leq 2$, at least after tensoring Hom groups with $\mathbf{Q}$, by [25, Th. 7.8.4 b)] .
} 
The second equality implies that $\operatorname{Ext}_{\mathcal{A}}^{1}\left(H^{0}(S), A\right)=0$, hence $H^{0}(S)$ is a projective object in $\mathcal{A}$.

Note that, then, $\operatorname{Ext}_{\mathcal{A}}^{i}\left(H^{0}(S), A\right)=0$ for all $i>0$. However, if $\mathcal{S}\left(H^{0}(S), A[i]\right)=0$ for all $i>0$ and the $t$-structure is non-degenerate, the same Yoneda argument shows that $S \stackrel{\sim}{\longrightarrow} H^{0}(S)[0]$.

This applies to show that the natural functor $D(\mathbf{H I}) \rightarrow \mathbf{D M}^{\text {eff }}$ is not full: take $S=\mathbf{Z}(2)[2]$, use Proposition 4.2 .1 below to see that $S$ is projective, and then the fact that $\mathcal{H}^{-1}(S) \simeq \mathcal{K}_{3}^{\text {ind }} \neq 0[35,38]$ to get a contradiction.

By Remark 3.6.4, the isomorphism $S \stackrel{\sim}{\longrightarrow} H^{0}(S)[0]$ also fails in general for $\mathcal{S}=\mathbf{D M}^{\circ}$ and $S=M^{\circ}(X), X$ smooth proper. In particular, the conservative functor $D\left(\mathbf{H I}^{\circ}\right) \rightarrow \mathbf{D M}^{\circ}$ of Theorem 3.2.2 $\mathrm{g}$ ) is not full.

\section{Further EXAMPLES OF BIRATIONAL SHEAVES}

Throughout this section, $F$ is supposed perfect.

4.1. Constant sheaves, abelian varieties and 0-cycles. All these are examples of birational sheaves. For the first ones, this is obvious. If $A$ is an abelian variety, then $X \mapsto A(X)$ defines an object of $\mathbf{H I}$ [3, Lemma 1.3.2], and this sheaf is birational by [39, Th. 3.1]. Finally, for any smooth proper variety $Y$, the assignment $X \mapsto C H_{0}\left(Y_{F(X)}\right)$ defines an object of $\mathbf{H I}^{\circ}$ by Theorem 2.1.2.

\subsection{Birational sheaves and contractions. Recall:}

4.2.1. Proposition ([31, Prop. 4.3 and Rk. 4.4]). The exact endofunctor $\mathcal{F} \mapsto \mathcal{F}_{-1}$ of $\mathbf{H I}$ (see $\left.\S 1.4\right)$ is given by the formulas

$$
\mathcal{F}_{-1}=\mathcal{H}^{0}\left(\underline{\operatorname{Hom}}_{\text {eff }}(\mathbf{Z}(1)[1], \mathcal{F}[0])\right)=\underline{\operatorname{Hom}}_{\mathbf{H I}}\left(\mathbb{G}_{m}, \mathcal{F}\right)
$$

where $\underline{\text { Hom }}_{\mathbf{H I}}$ is the internal Hom of the category HI. Moreover, $\underline{\operatorname{Hom}}_{\mathrm{eff}}(\mathbf{Z}(1)[1], \mathcal{F}[0])$ is acyclic in degrees $\neq 0$.

By this proposition, one has canonical isomorphisms for any $C \in$ $\mathrm{DM}^{\mathrm{eff}}$

$$
\mathcal{H}_{n}\left(\underline{\operatorname{Hom}}_{\text {eff }}(\mathbf{Z}(1)[1], C)\right) \simeq \mathcal{H}_{n}(C)_{-1} \quad(n \in \mathbf{Z}) .
$$

The next proposition gives some handle on the functor $i^{\mathrm{o}} \nu_{\leq 0}$. We shall use the following notation: for $\mathcal{F}, \mathcal{G} \in \mathbf{H I}$, we write

$$
\operatorname{Tor}_{i}^{\mathrm{DM}}(\mathcal{F}, \mathcal{G})=\mathcal{H}_{i}(\mathcal{F}[0] \otimes \mathcal{G}[0]), \quad \mathcal{F} \otimes_{\mathbf{H I}} \mathcal{G}=\operatorname{Tor}_{0}^{\mathrm{DM}}(\mathcal{F}, \mathcal{G})
$$

where the tensor product $\mathcal{F}[0] \otimes \mathcal{G}[0]$ is computed in $\mathbf{D} \mathbf{M}^{\text {eff }}$.

Note that $\otimes_{\mathbf{H I}}$ is the tensor product on $\mathbf{H I}$ induced by the one on $\mathbf{D M}^{\text {eff }}$; it is right $t$-exact because the tensor product in $D(\mathbf{P S T})$ is 
right $t$-exact and the functor $L C \circ L a: D(\mathbf{P S T}) \rightarrow \mathbf{D M}^{\text {eff }}$ is right $t$-exact. In particular, $\operatorname{Tor}_{i}^{\mathrm{DM}}(\mathcal{F}, \mathcal{G})=0$ for $i<0$. On the other hand, $\operatorname{Tor}_{i}^{\mathrm{DM}}(-,-)$ need not yield the $i$-th derived functor of $\otimes_{\mathbf{H I}}$ (assuming it exists), see $\S 3.7$.

Let $\mathcal{F} \in \mathbf{H I}$. By adjunction, Proposition 4.2.1 yields a map

$$
\varepsilon_{\mathcal{F}}: \mathbb{G}_{m} \otimes_{\text {HI }} \mathcal{F}_{-1} \rightarrow \mathcal{F} .
$$

4.2.2. Proposition. a) Let $C \in \mathbf{D M}^{\mathrm{eff}}$ be such that $\mathcal{H}_{q}(C)=0$ for $q<0$. Then $\mathcal{H}_{0}\left(i^{\mathrm{o}} \nu_{\leq 0} C\right)=$ Coker $\varepsilon_{\mathcal{H}_{0}(C)}$.

b) If $C$ is a sheaf $\mathcal{F}$ concentrated in degree 0 , we have

$$
\mathcal{H}_{q}\left(i^{\mathrm{o}} \nu_{\leq 0} \mathcal{F}[0]\right)= \begin{cases}\operatorname{Tor}_{q-1}^{\mathrm{DM}}\left(\mathbb{G}_{m}, \mathcal{F}_{-1}\right) & \text { for } q>1 \\ \operatorname{Ker} \varepsilon_{\mathcal{F}} & \text { for } q=1 \\ \operatorname{Coker} \varepsilon_{\mathcal{F}} & \text { for } q=0 \\ 0 & \text { for } q<0 .\end{cases}
$$

In particular, the sheaves on the right hand side belong to $\mathbf{H I}^{\circ}$.

Proof. Using Proposition 3.6.2, (4.1) gives a) for $n=0$ and b) if $C=$ $\mathcal{F}[0]$.

We can strengthen this proposition as follows, thus giving an interesting way to produce birational sheaves:

4.2.3. Proposition. Let $\mathcal{F} \in \mathbf{H I}$. Then

a) For all $q>0$, $\operatorname{Tor}_{q}^{\mathbf{D M}}\left(\mathbb{G}_{m}, \mathcal{F}\right) \in \mathbf{H I}^{\circ}$.

b) The adjunction map

$$
\mathcal{F} \rightarrow\left(\mathbb{G}_{m} \otimes_{\mathbf{H I}} \mathcal{F}\right)_{-1}
$$

is an isomorphism.

Proof. We shall prove a) and b) together. By quasi-invertibility of $\mathbf{Z}(1)$ [55], the adjunction map

$$
\mathcal{F} \rightarrow \underline{\operatorname{Hom}}_{\text {eff }}(\mathbf{Z}(1), \mathcal{F}(1))
$$

is an isomorphism. The right hand side may be rewritten $\underline{\operatorname{Hom}}_{\text {eff }}\left(\mathbb{G}_{m}, \mathbb{G}_{m}\right.$ $\otimes \mathcal{F})$. By Proposition 4.2.1, its $q$-th homology sheaf is $\operatorname{Tor}_{q}^{\text {DM }}\left(\mathbb{G}_{m}, \mathcal{F}\right)_{-1}$, which is therefore 0 for $q>0$. The conclusion now follows from Proposition 1.5.2. 
4.3. Birational sheaves and tensor products. In this subsection, we show that the tensor product in $\mathbf{H I}$ of two birational sheaves is birational.

More correctly, let $\nu_{0}: \mathbf{H I} \rightarrow \mathbf{H I}^{\mathrm{O}}$ be the left adjoint of $i^{\mathrm{O}}$ (Proposition 1.6.3): by Lemma A.3.1, $\nu_{0} \mathcal{F}=\mathcal{H}_{0}\left(\nu_{\leq 0} \mathcal{F}[0]\right)$. Then $\nu_{0}$ induces on $\mathbf{H I}^{\circ}$ a tensor structure $\otimes_{\mathbf{H I}^{\circ}}$ characterised by

$$
\nu_{0} \mathcal{F} \otimes_{\mathbf{H I}^{\circ}} \nu_{0} \mathcal{G}=\nu_{0}\left(\mathcal{F} \otimes_{\mathbf{H I}} \mathcal{G}\right)
$$

and this tensor product is right $t$-exact.

Let $\mathcal{F}, \mathcal{G} \in \mathbf{H I}^{\circ}$. From the isomorphism

$$
\nu_{0}\left(i^{\mathrm{o}} \mathcal{F} \otimes_{\mathbf{H I}} i^{\mathrm{o}} \mathcal{G}\right) \simeq \nu_{0} i^{\mathrm{o}} \mathcal{F} \otimes_{\mathbf{H I}^{\circ}} \nu_{0} i^{\mathrm{o}} \mathcal{G} \stackrel{\sim}{\longrightarrow} \mathcal{F} \otimes_{\mathbf{H I}^{\circ}} \mathcal{G}
$$

we get by adjunction a morphism

$$
i^{\mathrm{o}} \mathcal{F} \otimes_{\mathbf{H I}} i^{\mathrm{o}} \mathcal{G} \rightarrow i^{\mathrm{o}}\left(\mathcal{F} \otimes_{\mathbf{H I}^{\circ}} \mathcal{G}\right) .
$$

4.3.1. Theorem. (4.4) is an isomorphism.

The analogous result for the inclusion HI $\hookrightarrow$ NST is well-known to be false: for example $\mathbb{G}_{m} \otimes_{\mathbf{N S T}} \mathbb{G}_{m}$ is not homotopy invariant. Indeed, its quotient $\mathbb{G}_{m} \otimes_{\text {HI }} \mathbb{G}_{m}$ verifies $\mathbb{G}_{m} \otimes_{\text {HI }} \mathbb{G}_{m}(F)=K_{2}^{M}(F)$ [36, Th. 5.1], while $\mathbb{G}_{m} \otimes_{\text {NST }} \mathbb{G}_{m}(F)=F^{*} \otimes F^{*}$ if $F$ is algebraically closed.

Proof. For ease of notation, let us suppress the use of $i^{\circ}$. So we must show that the sheaf $\mathcal{H}=\mathcal{F} \otimes_{\mathbf{H I}} \mathcal{G}$ is birational. We shall use the characterisation of birational sheaves given in Proposition 1.5.1.

So, let $K / F$ be a function field, $C / K$ be a (proper) regular curve and $c \in C$ be a closed point. We must show that the map

$$
\mathcal{H}\left(\mathcal{O}_{C, c}\right) \rightarrow \mathcal{H}(K(C))
$$

is surjective.

By the surjectivity of the map in $[31,(2.10)]$, the composition

$$
\bigoplus_{[E: K(C)]<\infty} \mathcal{F}(E) \otimes \mathcal{G}(E) \rightarrow \bigoplus_{[E: K(C)]<\infty} \mathcal{H}(E) \stackrel{\left(T r_{E / K(C)}\right)}{\longrightarrow} \mathcal{H}(K(C))
$$

is surjective.

For each $E$ as above, let $C_{E}$ be the proper regular model of $E / K$; let $f_{E}: C_{E} \rightarrow C$ be the canonical map and let $c_{E}=f_{E}^{-1}(c)$. Then $\mathcal{O}_{C_{E}, c_{E}}$ is finite over $\mathcal{O}_{C, c}$, hence we have a commutative diagram

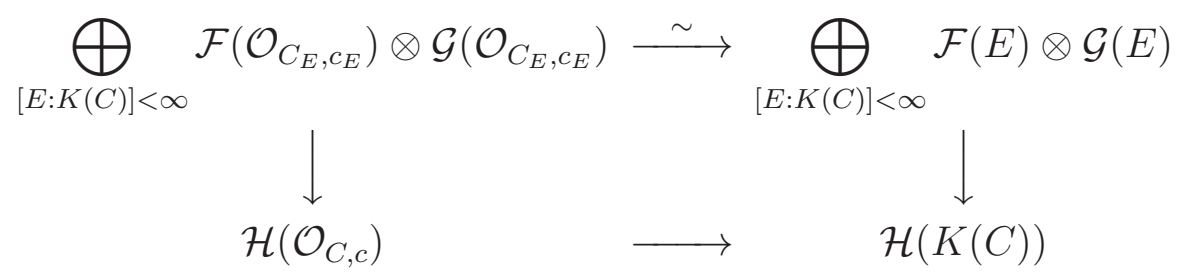


where the top map is an isomorphism because $\mathcal{F}$ and $\mathcal{G}$ are birational. The theorem follows.

4.3.2. Remark. Let $E$ be an elliptic curve: one can show that $\operatorname{Tor}_{1}^{\mathrm{DM}}(E, E)$ is not birational, see [30, Prop. 4.2]. Hence the functor $i^{\circ}: \mathbf{D M}^{\mathrm{o}} \rightarrow$ $\mathrm{DM}^{\mathrm{eff}}$ is not monoidal.

\section{Birational SheAves AND CYCle MOdUles}

Let CM denote the category of cycle modules in the sense of Rost [46]. In [29, Th. 6.2.3] we defined a pair of adjoint functors

$$
K^{?}: \text { Mod- } \text { Chow }^{\mathrm{O}} \leftrightarrows \mathbf{C M}: A^{0}
$$

where $A^{0}$ sends a cycle module $M=\left(M_{n}\right)_{n \in \mathbf{Z}}$ to a functor extending the assignment $X \mapsto A^{0}\left(X, M_{0}\right)$, and $K^{\text {? }}$ is fully faithful. The description of the essential image of $K^{\text {? }}$ was left open; we now have the material to answer this question.

5.1. Cycle modules and Somekawa $K$-groups. To formulate the answer, we need some preparation. Recall that, in [49], Somekawa associated an abelian group $K\left(F ; G_{1}, \ldots, G_{n}\right)$ to $n$ semi-abelian varieties $G_{1}, \ldots, G_{n}$ over $F$; this definition was extended in [31] to objects $G_{1}, \ldots, G_{n}$ of $\mathbf{H I}{ }^{7}$

Let $M$ be a cycle module. Recall now that Déglise associated to $M$ a graded object $\left(\mathcal{M}_{n}\right)$ of $\mathbf{H I}$ such that

$$
\mathcal{M}_{n}(X)=A^{0}\left(X, M_{n}\right)
$$

for any $X \in \mathbf{S m}$. We then have:

5.1.1. Lemma. For any extension $E / F$, the map $E^{*} \otimes M_{n}(E) \rightarrow$ $M_{n+1}(E)$ from $[46$, D3] induces a homomorphism

$$
\theta_{n}: K\left(E ; \mathbb{G}_{m}, \mathcal{M}_{n}\right) \rightarrow M_{n+1}(E) .
$$

Proof. Recall from [31, Def. 5.1] that $K\left(E ; \mathbb{G}_{m}, \mathcal{M}_{n}\right)$ has generators $\{f, m\}_{E^{\prime} / E}$ where $E^{\prime}$ runs through the finite extensions of $E, f \in E^{*}$ and $m \in \mathcal{M}_{n}(\operatorname{Spec} E)=M_{n}(E)$, subject to 3 types of relations directly generalising those of $[49,(1.2 .0),(1.2 .1)$ and $(1.2 .2)]$ : bilinearity, the projection formula and relations "of Somekawa type". Write $\varphi: E \rightarrow$ $E^{\prime}$ for the inclusion. We define $\theta_{n}(\{m, f\})=\varphi^{*}(f \cdot m)$, where $\varphi^{*}$ is the transfer map from [46, D2] and the $f \cdot m$ is the product from [46, D3]. We need to check that $\theta_{n}$ respects the relations. Bilinearity is obvious and the projection formula follows from [46, R2b and R2c]. By [31,

\footnotetext{
${ }^{7}$ Recall that a semi-abelian variety defines an object of $\mathbf{H I}$ by [48, Lemma 3.2] and [3, Lemma 1.3.2].
} 
Rk. 6.3], to prove the relations of Somekawa type it now suffices to prove the finer relations "of geometric type" from loc. cit., Def. 6.1; these follow directly from [46, (RC) in Prop. 2.2].

5.1.2. Theorem. Let $M \in \mathbf{C M}$. Then $M$ is in the essential image of $K^{?}$ if and only if:

(i) $M_{n}=0$ for $n<0$;

(ii) for any $n \geq 0$, the map $\theta_{n}$ of Lemma 5.1.1 is an isomorphism.

5.2. A reformulation. We first translate Theorem 5.1.2 into a statement involving homotopy invariant Nisnevich sheaves with transfers, whose proof is then rather straightforward. For this, we need to recall Déglise's theory from [12] in further detail:

By a construction going back to Morel and Voevodsky, the category $\mathrm{DM}^{\mathrm{eff}}$ can be fully embedded into a larger $\otimes$-triangulated category DM of "Z(1)-spectra". There is an adjunction [12, Prop. 4.7]:

$$
\Sigma^{\infty}: \mathrm{DM}^{\mathrm{eff}} \leftrightarrows \mathrm{DM}: \Omega^{\infty}
$$

where $\Sigma^{\infty}$ is fully faithful, monoidal and $\Sigma^{\infty} \mathbf{Z}(1)$ is invertible. Moreover the homotopy $t$-structure of $\mathbf{D M}^{\text {eff }}$ extends to a $t$-structure on DM, with heart the category $\mathbf{H I}_{*}$ of homotopic modules [12, Th. 5.11]; the functor $\Omega^{\infty}$ is $t$-exact.

By [12, Def. 1.17], an object of $\mathbf{H I}_{*}$ is a sequence $\left(\mathcal{M}_{n}, \varepsilon_{n}\right)_{n \in \mathbf{Z}}$, where $\mathcal{M}_{n} \in \mathbf{H I}$ and $\varepsilon_{n}$ is an isomorphism $\mathcal{M}_{n} \stackrel{\sim}{\longrightarrow} \underline{\operatorname{Hom}}_{\mathbf{H I}}\left(\mathbb{G}_{m}, \mathcal{M}_{n+1}\right)$. By Proposition 4.2.1, this may also be written $\mathcal{M}_{n} \stackrel{\sim}{\longrightarrow}\left(\mathcal{M}_{n+1}\right)_{-1} .{ }^{8}$ Morphisms are given componentwise. The adjoint functors

$$
\sigma^{\infty}: \mathbf{H I} \leftrightarrows \mathbf{H I}_{*}: \omega^{\infty}
$$

induced by $\Omega^{\infty}$ and $\Sigma^{\infty}$ are given by

$$
\begin{aligned}
\omega^{\infty} \mathcal{M}_{*} & =\mathcal{M}_{0} \\
\left(\sigma^{\infty} \mathcal{F}\right)_{n} & = \begin{cases}\mathcal{F} \otimes_{\mathbf{H I}} \mathbb{G}_{m}^{\otimes n} & \text { if } n \geq 0 \\
\mathcal{F}_{n} \text { (Voevodsky's contraction) } & \text { if } n<0 .\end{cases}
\end{aligned}
$$

Déglise's main result, [12, Th. 3.7], provides an equivalence of categories between $\mathbf{H I}_{*}$ and $\mathbf{C M}$. More precisely, his functor $\mathbf{C M} \rightarrow \mathbf{H I}_{*}$ sends a cycle module $M_{*}$ to a homotopic module $\mathcal{M}_{*}$ given by (5.1). His functor $\mathbf{H I}_{*} \rightarrow \mathbf{C M}$ sends a homotopic module $\mathcal{M}_{*}$ to a cycle module $M_{*}$ such that

$$
M_{n}(K)=\lim _{\longrightarrow} \mathcal{M}_{n}(U)
$$

\footnotetext{
${ }^{8}$ In [12], Déglise writes $S_{t}^{1}$ for $\mathbb{G}_{m}$. These sheaves are isomorphic, as follows for example from [53, Th. 3.4.2 (iii)] applied to $C=\mathbf{A}^{1}-\{0\}$.
} 
for any function field $K / F$, where $U$ runs through the collection of smooth models of $K$ and transition maps are open immersions [12, 2.10 and 3.1].

On the other hand, the full embedding $D: \mathbf{C o r}_{\text {rat }}^{\circ} \rightarrow$ BFC of Proposition 2.3.3 yields a pair of adjoint functors

$$
D_{!}: \operatorname{Mod}-\mathrm{Chow}^{\mathrm{o}} \leftrightarrows \mathrm{HI}^{\mathrm{o}}: D^{*}
$$

Recall finally that by $[31,(1.1)]$, we have an isomorphism

$$
K\left(E ; \mathbb{G}_{m}, \mathcal{M}_{n}\right) \simeq\left(\mathbb{G}_{m} \otimes_{\mathbf{H I}} \mathcal{M}_{n}\right)(\operatorname{Spec} E)
$$

where $\mathcal{M}_{n}$ is associated to a given cycle module $M$ as in (5.1). The translation we need for proving Theorem 5.1.2 is now provided by:

5.2.1. Proposition. The functors $K^{\text {? }}$ and $A^{0}$ are respectively isomorphic to the compositions $\sigma^{\infty} \circ i^{\circ} \circ D_{!}$and $D^{*} \circ R_{\mathrm{nr}}^{0} \circ \omega^{\infty}$.

Proof. By adjunction, it suffices to construct a natural isomorphism $K^{?} \simeq \sigma^{\infty} \circ i^{\mathrm{o}} \circ D_{!}$. By construction, $K^{?}$ is obtained as the left Kan extension of Merkurjev's functor

$$
X \mapsto K^{X}
$$

where, for a smooth projective variety $X, K^{X}$ is a cycle module such that $K_{*}^{X}(E)=A_{0}\left(X_{E}, K_{*}\right)$ for any function field $E / F$. Here $K$ denotes the cycle module given by Milnor $K$-theory. As $K^{X}$ represents the functor $M_{*} \mapsto A^{0}\left(X, M_{0}\right)$ [37, Th. 2.10], its image in $\mathbf{H I}_{*}$ represents the functor $\mathcal{M}_{*} \mapsto H_{\mathrm{Nis}}^{0}\left(X, \mathcal{M}_{0}\right)$. But

$$
\begin{aligned}
H^{0}\left(X, \mathcal{M}_{0}\right) & \simeq \operatorname{Hom}_{\mathbf{H I}}\left(h_{0}^{\mathrm{Nis}}(X), \mathcal{M}_{0}\right) \\
& =\operatorname{Hom}_{\mathbf{H I}}\left(h_{0}^{\mathrm{Nis}}(X), \omega^{\infty} \mathcal{M}_{*}\right) \simeq \operatorname{Hom}_{\mathbf{H I}}\left(\sigma^{\infty} h_{0}^{\mathrm{Nis}}(X), \mathcal{M}_{*}\right)
\end{aligned}
$$

so that the image of $K^{X}$ in $\mathbf{H I}_{*}$ is $\sigma^{\infty} h_{0}^{\mathrm{Nis}}(X)$. Since $D_{!} y\left(h^{\mathrm{o}}(X)\right)=$ $h_{0}^{\mathrm{Nis}}(X)$ by Corollary 2.3.4, this concludes the proof.

5.3. Proof of Theorem 5.1.2. In view of Proposition 5.2.1, we have to show that the essential image of the composite functor $\mathbf{H I}^{\mathrm{o}} \stackrel{i^{\circ}}{\longrightarrow}$ HI $\stackrel{\sigma^{\infty}}{\longrightarrow} \mathbf{H I}_{*}$ consists of those homotopic modules $\mathcal{F}_{*}$ such that

(i) $\mathcal{F}_{n}=0$ for $n<0$;

(ii) for any $n \geq 0$, the canonical map $\mathcal{F}_{0} \otimes_{\mathbf{H I}} \mathbb{G}_{m}^{\otimes n} \rightarrow \mathcal{F}_{n}$ is an isomorphism.

Any homotopic module in the essential image if $\sigma^{\infty} i^{\circ}$ verifies (i) by Proposition 1.5.2 and (ii) by the above description of $\sigma^{\infty}$. For the converse, we use the fact that $i^{\mathrm{o}}$ has a right adjoint $R_{\mathrm{nr}}^{0}$ (Proposition 
1.6.3). Let $\mathcal{F}_{*} \in \mathbf{H I}_{*}$ verify (i) and (ii): we must show that the counit map

$$
\sigma^{\infty} i^{\mathrm{o}} R_{\mathrm{nr}}^{0} \omega^{\infty} \mathcal{F}_{*} \rightarrow \mathcal{F}_{*}
$$

is an isomorphism. We have $\omega^{\infty} \mathcal{F}_{*}=\mathcal{F}_{0}$; by Proposition 1.5.2, $\mathcal{F}_{0} \in$ $\mathbf{H I}^{\mathrm{o}}$, hence $i^{\mathrm{o}} R_{\mathrm{nr}}^{0} \mathcal{F}_{0}=\mathcal{F}_{0}$ and the claim.

\section{UNRAMIFIED COHOMOLOGY}

6.1. The functor $R_{\mathrm{nr}}$. In this section, we assume $F$ perfect. We study here the right adjoint to $i^{\mathrm{o}}: \mathbf{D M}^{\mathrm{o}} \rightarrow \mathbf{D M}^{\text {eff }}$ from Theorem 3.3.5: this right adjoint is denoted by $R_{\mathrm{nr}}$.

For any $C \in \mathbf{D M}^{\text {eff }}$ and $q \in \mathbf{Z}$, we write $R_{\mathrm{nr}}^{q} C$ for $\mathcal{H}^{q}\left(R_{\mathrm{nr}} C\right) \in$ $\mathbf{H I}^{\mathrm{o}}$, where $\mathcal{H}^{q}$ corresponds to the homotopy $t$-structure on $\mathbf{D M}^{\mathrm{o}}$. By Theorem 3.4.1 and Lemma A.3.1, $R_{\mathrm{nr}}$ is left exact with respect to the homotopy $t$-structures of $\mathbf{D M}^{\text {eff }}$ and $\mathbf{D M}^{\circ}$. In particular, $R_{\mathrm{nr}}^{q} \mathcal{F}=0$ for $q<0$ if $\mathcal{F} \in \mathbf{H I}$, and Lemma A.3.1 shows that the functor

$$
\mathcal{F} \mapsto R_{\text {nr }}^{0} \mathcal{F}
$$

from $\mathbf{H I}$ to $\mathbf{H I}^{\mathrm{o}}$ is the right adjoint to the inclusion functor $i^{\mathrm{o}}: \mathbf{H I}^{\mathrm{o}} \rightarrow$ HI from Proposition 1.6.3.

The main result of this section is Theorem 6.3.1: for any $\mathcal{F} \in \mathbf{H I}$, $R_{\mathrm{nr}}^{0} \mathcal{F}$ coincides with the unramified part of $\mathcal{F}$ in the sense of classical unramified cohomology [10].

See [30] for computations of the "higher derived functors of unramified cohomology" $R_{\mathrm{nr}}^{q} \mathcal{F}$, for $q>0$ and certain $\mathcal{F}$ 's.

\subsection{The group $\mathcal{F}_{\mathrm{nr}}(X)$.}

6.2.1. Definition. Let $K / F$ be a finitely generated field extension.

a) A valuation $v$ on $K$ (trivial on $F$ ) is divisorial if it is discrete of rank 1 and its valuation ring is of the form $O_{V, x}$, for $V$ a smooth $F$-scheme of finite type with function field $K$. (These are the prime divisors of Zariski-Samuel, [56, Ch. VI, §14].)

b) For $\mathcal{F} \in \mathbf{H I}$, we set

$$
\mathcal{F}_{\mathrm{nr}}(K / F)=\operatorname{Ker}\left(\mathcal{F}(K) \stackrel{\left(\partial_{v}\right)}{\longrightarrow} \prod_{v} \mathcal{F}_{-1}(F(v))\right)
$$

where $v$ runs through all the divisorial valuations on $K$.

c) If $X$ is a smooth model of $K$, we set $\mathcal{F}_{\mathrm{nr}}(X)=\mathcal{F}_{\mathrm{nr}}(K / F)$.

The exact sequence (1.6) implies:

6.2.2. Lemma. For any $X \in \mathbf{S m}, \mathcal{F}_{\mathrm{nr}}(X) \subseteq \mathcal{F}(X)$. 
We are going to show that $\mathcal{F}_{\text {nr }}$ is preserved by finite correspondences. We begin with a series of lemmas.

6.2.3. Lemma. Let $f: Y \rightarrow X$ be a dominant morphism of smooth irreducible varieties. Then $f^{*} \mathcal{F}_{\mathrm{nr}}(X) \subset \mathcal{F}_{\mathrm{nr}}(Y)$.

Proof. Let $\alpha \in \mathcal{F}_{\mathrm{nr}}(X)$, and let $w$ be a divisorial valuation on the function field $L$ of $Y$. Since $f$ is dominant, $L$ is an extension of $K$. Let $v$ be the restriction of $w$ to $K$. Then

$$
\partial_{w}\left(f^{*} \alpha\right)=e f^{*} \partial_{v}(\alpha)
$$

where $e$ is the ramification index if $v$ is nontrivial and $e=0$ if $v$ is trivial; we simply write $f: \operatorname{Spec} F(w) \rightarrow \operatorname{Spec} F(v)$ for the map induced by $f$. This formula follows from examining the purity isomorphism of $[52$, Lemma 4.36]. It also follows from Déglise's theory of generic motives $[11$, lemme 5.4.7], since the residues $\partial$ may be expressed in their terms $[12,3.1]$.

6.2.4. Lemma. Let $f: X \rightarrow Y$ be a finite surjective morphism of smooth F-varieties. Let ${ }^{t} f \in c(Y, X)$ be the transpose of its graph. Then $\left({ }^{t} f\right)^{*} \mathcal{F}_{\mathrm{nr}}(X) \subseteq \mathcal{F}_{\mathrm{nr}}(Y)$.

Proof. Here, $K=F(X)$ is a finite extension of $L=F(Y)$. Let $\alpha \in$ $\mathcal{F}_{\mathrm{nr}}(X)$, and let $v$ be a divisorial valuation of $L$. Then

$$
\partial_{v}\left(\left({ }^{t} f\right)^{*} \alpha\right)=\sum_{w \mid v}\left({ }^{t} f\right)^{*} \partial_{w}(\alpha)
$$

[11, lemme 5.4.7].

We need the following version of a theorem of Knaf-Kuhlmann [34, Th. 1.1]. ${ }^{9}$

6.2.5. Proposition. Let $K / F$ be a function field, $w$ a divisorial valuation of $K$ with residue field $L, v$ a divisorial valuation of $L$. Then there is a closed immersion $i: Z \hookrightarrow W$ of smooth $F$-varieties such that $K \simeq F(W), L \simeq F(Z), \mathcal{O}_{W, Z} \simeq \mathcal{O}_{w}$, and $v$ is finite on $Z$.

Proof. Let $u$ be the composite valuation, which is discrete of rank 2 (here we identify valuations with the associated surjective places): it is an Abyankhar place in the sense of [34]. The residue field of $u$ is separably generated since $F$ is perfect. Let $\mathcal{O}_{u} \subset K$ be the local ring of $u$ and $a, b \in \mathcal{O}_{u}$ be two elements such that $u(a)=(1,0)$ and $u(b)=(0,1)$. By [34, Th. 1.1], there is a smooth model $W$ of $K$ on

\footnotetext{
${ }^{9}$ Its proof obviously extends to compositions of more than 2 divisorial valuations.
} 
which $u$ has a centre $t$ of codimension 2 , and such that $a, b$ are $\mathcal{O}_{W, t^{-}}$ monomials in $a_{1}, a_{2}$ (in the sense of [34, p. 234]) for some regular system of parameters $\left(a_{1}, a_{2}\right)$ of $\mathcal{O}_{W, t}$. This easily implies that $u\left(a_{1}\right)=(1,0)$ and $u\left(a_{2}\right)=(0,1)$, up to permuting $\left(a_{1}, a_{2}\right)$. Then $\mathcal{O}_{W, t} / a_{1} \mathcal{O}_{X, t}$ is regular [56, Ch. VIII, $\S 11$, Th. 26], and is the local ring of $t$ on $Z$, the closure of the centre of $w$ on $W$. Thus $Z$ is regular at $t$, hence smooth around $t$ since $F$ is perfect, and we can make it smooth by shrinking $W$.

6.2.6. Lemma (Main lemma). Let $i: Y \rightarrow X$ be a closed immersion of codimension 1 . Then $i^{*} \mathcal{F}_{\mathrm{nr}}(X) \subseteq \mathcal{F}_{\mathrm{nr}}(Y)$.

Proof. Let $v$ be a divisorial valuation of $F(Y)$, and let $w$ be the divisorial valuation on $K=F(X)$ defined by $i: Y \rightarrow X$. Let $u$ be the composite valuation, and let $(W, Z)$ be as in the conclusion of Proposition 6.2.5. We thus have two closed immersions of codimension 1:

$$
\begin{aligned}
i: Y & \rightarrow X \\
i^{\prime}: Z & \rightarrow W .
\end{aligned}
$$

Since $w$ is finite on $X$ and $W$, they share an open neighbourhood $U$ of Spec $O_{w}$. Let $T$ be the closure of the centre of $w$ in $U$. Since $Y, Z$ and $T$ are birational, they share a nonempty open subset $U^{\prime}$. We now have the following situation:

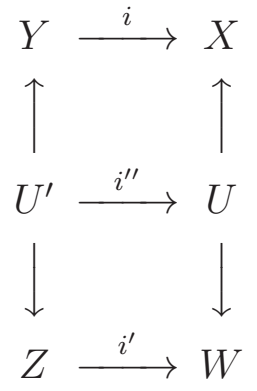

where all varieties are smooth, vertical maps are open immersions, $i$ and $i^{\prime}$ are closed immersions while $i^{\prime \prime}$ is locally closed. This in turn gives a commutative diagram

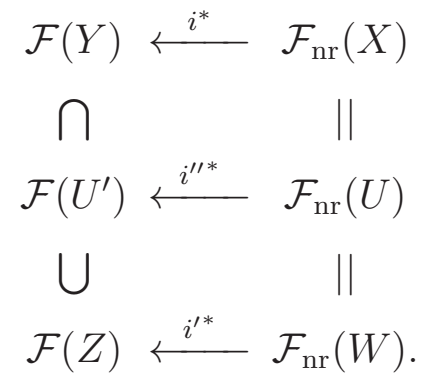


Here we identified the three left-hand terms with subgroups of $\mathcal{F}(L)$ thanks to (1.6), where $L=F(Y)$. Let $\alpha \in \mathcal{F}_{\mathrm{nr}}(X)$. The diagram shows that $i^{*} \alpha \in \mathcal{F}(Y)$ lies in $\mathcal{F}(Z)$. Since $v$ is finite on $Z$, we have $\partial_{v}\left(i^{*} \alpha\right)=0$ by (1.6) applied to $Z$. Since $v$ was an arbitrary divisorial valuation of $L$, this shows that $i^{*} \alpha$ is unramified, as requested.

6.3. $\mathcal{F}_{\mathrm{nr}}$ and $R_{\mathrm{nr}}^{0} \mathcal{F}$. The following theorem justifies the notation $R_{\mathrm{nr}} \mathcal{F}$ :

6.3.1. Theorem. Let $\mathcal{F} \in \mathbf{H I}$. Let $X \in \mathbf{S m}$ be irreducible with function field $K$. Then there is a natural isomorphism

$$
R_{\mathrm{nr}}^{0} \mathcal{F}(X)=\mathcal{F}_{\mathrm{nr}}(X) .
$$

Proof. Recall that, by Proposition 1.6.3, the counit map

$$
i^{\mathrm{o}} R_{\mathrm{nr}}^{0} \mathcal{F} \rightarrow \mathcal{F}
$$

is a monomorphism. By Lemma 6.2.2, we may thus identify both groups with subgroups of $\mathcal{F}(X)$. We proceed in two steps:

(1) $R_{\mathrm{nr}}^{0} \mathcal{F}(X) \subset \mathcal{F}_{\mathrm{nr}}(X)$.

(2) $X \mapsto \mathcal{F}_{\mathrm{nr}}(X)$ defines an object of $\mathbf{H I}^{\mathrm{O}}$.

Granting (1) and (2), the theorem follows from the universal property of $R_{\mathrm{nr}}^{0} \mathcal{F}$.

(1) Let $\alpha \in R_{\mathrm{nr}}^{0} \mathcal{F}(X)$, and let $v$ be a divisorial valuation of $K$. We want to show that $\partial_{v}(\alpha)=0$. Choose a smooth model $V$ of $K$ on which $v$ is finite, with centre a point $x$ of codimension 1 . Let $U$ be a common open subset of $X$ and $V$. Since $R_{\mathrm{nr}}^{0} \mathcal{F} \in \mathbf{H I}^{\circ}$, we have isomorphisms

$$
R_{\mathrm{nr}}^{0} \mathcal{F}(X) \stackrel{\sim}{\longleftarrow} R_{\mathrm{nr}}^{0} \mathcal{F}(U) \stackrel{\sim}{\longrightarrow} R_{\mathrm{nr}}^{0} \mathcal{F}(V) \subset \mathcal{F}(V)
$$

and the claim follows from the complex (1.6).

(2) By Proposition 1.3.3 a), it suffices to show that $\mathcal{F}_{\mathrm{nr}}$ defines a sub-presheaf with transfers of $\mathcal{F}$. Let $\varphi \in c(Y, X)$ be a finite correspondence, with $Y$ smooth irreducible. We have to show that $\varphi^{*} \mathcal{F}_{\text {nr }}(X) \subseteq$ $\mathcal{F}_{\mathrm{nr}}(Y)$. For this, we may assume that $\varphi$ is defined by an irreducible subset $Z \subset Y \times X$.

Let $p: Z \rightarrow Y$ be the projection. There is a nonempty open subset $U \subseteq Y$ such that $p^{-1}(U)$ is smooth. The transpose of the graph of $p_{\mid p^{-1}(U)}$ defines a finite correspondence ${ }^{t} p \in c\left(U, p^{-1}(U)\right)$. Let $k$ be the immersion $p^{-1}(U) \rightarrow Z \rightarrow Y \times X$ and $\gamma_{k}: p^{-1}(U) \rightarrow p^{-1}(U) \times Y \times X$ 
be the associated graph map. Then the diagram

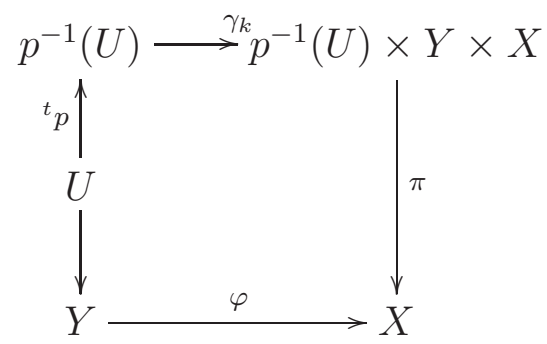

commutes in SmCor. Note that $\gamma_{k}$ is a regular embedding, hence may be locally written as a composition of closed embeddings of smooth varieties, of relative dimension 1. Lemmas 6.2.3, 6.2.6 and 6.2.4 then respectively show that $\pi^{*}, \gamma_{k}^{*}$ and $\left({ }^{t} p\right)^{*}$ respect unramified elements, and thus so does $\varphi^{*}$.

6.3.2. Corollary. Let $\mathcal{F} \in \mathbf{H I}$. Suppose that a smooth variety $X$ has a smooth compactification $\bar{X}$. Then $R_{\mathrm{nr}}^{0} \mathcal{F}(X)$ is given by the formula

$$
R_{\mathrm{nr}}^{0} \mathcal{F}(X)=\mathcal{F}(\bar{X}) \text {. }
$$

Proof. By Theorem 6.3.1, we may replace $R_{\mathrm{nr}}^{0} \mathcal{F}(X)$ by $\mathcal{F}_{\mathrm{nr}}(X)$; the conclusion is then classical [10, Prop. 2.1.8 e)]. Note that the "codimension 1 purity" hypothesis is satisfied in view of Theorem 1.4.2.

\section{Appendix A. Localisation, Brown Representability And $t$-STRUCTURES}

In this appendix, we collect technical results on triangulated categories which are used in the main body of the paper. §A.2 recollects results of Neeman on compactly generated triangulated categories $[41,42]$, also revisited by Beilinson-Vologodsky [6]; the existence of the functor $R_{\mathrm{nr}}$ rests on Theorem A.2.6 (v). §A.3 recalls the behaviour of $t$-structures under adjoints and localisations. The main result is Theorem A.4.2, from which Theorem 3.2.2 is deduced almost directly.

A.1. Terminology. It is worthwhile to first recall and fix some terminology on triangulated categories: we follow Neeman in [42]. As has become widespread, we replace the old terminology "exact functor" by "triangulated functor", and "distinguished triangle" by "exact triangle".

Let $\mathcal{T}$ be a triangulated category. Recall that a strictly full subcategory $\mathcal{S}$ of $\mathcal{T}$ is triangulated if it is additive and closed under the formation of shifts and cones. Then one can define the Verdier quotient 
$\mathcal{T} / \mathcal{S}$ [51]: this is a triangulated category which comes with a triangulated functor $\mathcal{T} \rightarrow \mathcal{T} / \mathcal{S}$, universal among triangulated functors sending all objects of $\mathcal{S}$ to 0 [51, Cor. II.2.2.11 c)]. We say that a triangulated functor $T: \mathcal{T} \rightarrow \mathcal{U}$ is a localisation if it induces an equivalence of categories $\mathcal{T} / T^{-1}(0) \stackrel{\sim}{\longrightarrow} \mathcal{U}$. A triangulated subcategory $\mathcal{S}$ of $\mathcal{T}$ is thick (resp. localising) if it is stable under representable direct summands (resp. and under representable direct sums). If $\mathcal{S}^{\natural} \subseteq \mathcal{T}$ is the smallest thick subcategory of $\mathcal{T}$ containing $\mathcal{S}$, the functor $\mathcal{T} / \mathcal{S} \rightarrow \mathcal{T} / \mathcal{S}^{\natural}$ is an equivalence of categories [51, Cor. II.2.2.11 a)]. We have:

A.1.1. Lemma ([42, Cor. 3.2.11]). Let $\mathcal{T}$ be a triangulated category with small direct sums. Let $\mathcal{S}$ be a localising subcategory. Then $\mathcal{T} / \mathcal{S}$ has small direct sums, and the universal functor $\mathcal{T} \rightarrow \mathcal{T} / \mathcal{S}$ preserves coproducts.

Recall that an object $X$ of $\mathcal{T}$ is compact if $\mathcal{T}(X,-)$ commutes with representable direct sums. A triangulated functor $T: \mathcal{T} \rightarrow \mathcal{T}^{\prime}$ is dense if the image of $T$ generates $\mathcal{T}^{\prime}$ in the sense that $\mathcal{T}^{\prime}$ is the smallest localising subcategory of itself that contains this image: this is the same notion as in [53].

A.1.2. Definition. Let $B$ be a class of objects in $\mathcal{T}$. We write

$$
\begin{aligned}
& B^{\perp}=\{X \in \mathcal{T} \mid \mathcal{T}(B[i], X)=0 \forall i \in \mathbf{Z}\} \\
& { }^{\perp} B=\{X \in \mathcal{T} \mid \mathcal{T}(X, B[i])=0 \forall i \in \mathbf{Z}\} .
\end{aligned}
$$

These are triangulated subcategories of $\mathcal{T}$. We also write

- $\langle B\rangle$ for the triangulated subcategory of $\mathcal{T}$ generated by $B$ (i.e. the smallest triangulated subcategory of $\mathcal{T}$ which contains $B$ );

- $\langle B\rangle^{\natural}$ for the thick subcategory of $\mathcal{T}$ generated by $B$;

- $\langle B\rangle^{\oplus}$ for the localising subcategory of $\mathcal{T}$ generated by $B$.

A.2. Some results of Neeman. Below we shall use results from Amnon Neeman's book [42], especially loc. cit., Lemma 4.4.5 and Theorem 4.4.9. Most of them are stated there with respect to an infinite cardinal $\alpha$; here, we shall only need the case where $\alpha=\aleph_{0}$ as in [41]. For the reader's convenience, we now state these results in this special case.

A.2.1. Theorem ([42, Th. 4.3.3 and Cor. 4.4.5]). Let $\mathcal{T}$ be a triangulated category with small direct sums, $\mathcal{T}^{c}$ its (thick) subcategory of compact objects, $S$ a thick subcategory of $\mathcal{T}^{c}$ and $\langle S\rangle^{\oplus}$ the localising subcategory of $\mathcal{T}$ generated by $S$. Let $(x, z) \in \mathcal{T}^{c} \times\langle S\rangle^{\oplus}$, and let $f \in \mathcal{T}(x, z)$. Then $f$ factors through an object of $S$. In particular, $\langle S\rangle^{\oplus}=\mathcal{T} \Rightarrow S=\mathcal{T}^{c}$. 
Let us sketch the proof: "in particular" is obtained by applying the theorem to the identity map of a compact object. The proof of A.2.1 goes as follows: Neeman introduces the full subcategory $\mathcal{S}$ of $\mathcal{T}$ consisting of those objects $z$ for which the conclusion of the theorem is valid for any compact $x$. He successively proves that $\mathcal{S}$ contains $S$, is triangulated and is closed under coproducts. Therefore $\mathcal{S}$ contains $\langle S\rangle^{\oplus}$.

This theorem is used in the proof of Proposition A.4.1 below.

A.2.2. Theorem ([42, Th. 4.4.9]). Let $\mathcal{S}$ be a compactly generated triangulated category with small direct sums, and let $R$ be a set of objects of $\mathcal{S}^{c}$. Write $\mathcal{R}$ for the localising subcategory generated by $R$. Then:

(i) $\mathcal{R}^{c}=\mathcal{R} \cap \mathcal{S}^{c}$. In particular, $\mathcal{R}=\left\langle\mathcal{R}^{c}\right\rangle^{\oplus}$.

(ii) The natural functor $\mathcal{S}^{c} / \mathcal{R}^{c} \rightarrow \mathcal{S} / \mathcal{R}$ factors through a full embedding $\mathcal{S}^{c} / \mathcal{R}^{c} \rightarrow(\mathcal{S} / \mathcal{R})^{c}$.

(iii) Any object of $(\mathcal{S} / \mathcal{R})^{c}$ is isomorphic to a direct summand of an object of $\mathcal{S}^{c} / \mathcal{R}^{c}$.

The next result we shall use is Neeman's "Brown representability theorem", which gives sufficient conditions for the existence of a right adjoint.

A.2.3. Definition ([42, Def. 8.2.1]). Let $\mathcal{T}$ be a triangulated category. We say that $\mathcal{T}$ has the Brown representability property if

(1) It has small direct sums.

(2) Any homological functor $H: \mathcal{T}^{\mathrm{op}} \rightarrow \mathbf{A b}$ which converts infinite direct sums into products is representable.

A.2.4. Lemma. a) Any adjoint (left or right) of a triangulated functor is triangulated.

b) Suppose that $\mathcal{T}$ has the Brown representability property. Let $f$ : $\mathcal{T} \rightarrow \mathcal{U}$ be a triangulated functor. Then $f$ has a right adjoint if and only if it commutes with infinite direct sums.

Proof. a) is proven in [42, Lemma 5.3.6]. We give the proof of b) since it is very simple. If $f$ has a right adjoint, it commutes with all representable colimits. Conversely, let $U \in \mathcal{U}$. We must prove that the functor $T \mapsto \mathcal{U}(f T, U)$ is representable. But if $f$ commutes with infinite direct sums, this functor converts infinite direct sums into products.

A.2.5. Theorem ([42, Prop. 8.4.2]). If $\mathcal{T}$ has small direct sums and is compactly generated, it has the Brown representability property.

We now get the following complement to Theorem A.2.2: 
A.2.6. Theorem. With the assumptions and notation of Theorem A.2.2,

(i) The localisation functor $\mathcal{S} \stackrel{\pi}{\longrightarrow} \mathcal{S} / \mathcal{R}$ has a right adjoint $j$.

(ii) The essential image of $j$ is $\mathcal{R}^{\perp}$.

(iii) Let $i: \mathcal{R} \rightarrow \mathcal{S}$ be the inclusion functor. Then $i$ has a right adjoint $p$, and for any object $X \in \mathcal{S}$, the sequence

$$
i p X \rightarrow X \rightarrow j \pi X
$$

defines an exact triangle.

(iv) $\left\langle(\mathcal{S} / \mathcal{R})^{c}\right\rangle^{\oplus}=\mathcal{S} / \mathcal{R}$.

(v) The functor $j$ itself has a right adjoint.

Proof. By assumption, $\mathcal{R}$ has small direct sums. It is compactly generated by Theorem A.2.2. Hence it has the Brown representability property by Theorem A.2.5. Lemma A.2.4 now implies that the functor $i$ of (iii) has a right adjoint.

Given this, assertions (i), (ii) and (iii) are part of a general theorem of Verdier [51, Prop. 2.3.3].

For (iv), we have $\mathcal{S} / \mathcal{R}=\left(\mathcal{S}^{c} / \mathcal{R}^{c}\right)^{\oplus}$ since $\mathcal{S}=\left(\mathcal{S}^{c}\right)^{\oplus}$ and $\pi$ commutes with small direct sums (Lemma A.1.1), and we conclude by Theorem A.2.2 (iii).

For $(\mathrm{v})$, let $j^{\prime}: \mathcal{R}^{\perp} \rightarrow \mathcal{S}$ be the inclusion. Observe that $\mathcal{R}^{\perp}=\left(\mathcal{R}^{c}\right)^{\perp}$ by denseness; by Theorem A.2.2 (i) this implies that $j^{\prime}$ commutes with small direct sums, hence, by (ii), so does $j$. Since, by (iv), $\mathcal{S} / \mathcal{R}$ is compactly generated, it has the Brown representability property which guarantees that $j$ has a right adjoint by Lemma A.2.4 b).

A.2.7. Remark. The proposition p. 1714 of Beilinson-Vologodsky [6] wraps up all the above, except for the existence of the right adjoint to $j$ in Theorem A.2.6 (v). It adds a nice explicit description of the objects of $\mathcal{R}$ : every such object can be represented as $\operatorname{hocolim}\left(M_{a}, i_{a}\right)$ where $M_{0}$ and each cone $\left(i_{a}\right)$ is a direct sum of translations of objects from $R$. We shall not use this result here.

A.3. Localisation and $t$-structures. The standard reference for $t$ structures is [5], whose notations we follow. We shall mainly use the following lemma [5, Prop. 1.3.17 (i), (iii)]:

A.3.1. Lemma. Let $F^{*}: \mathcal{S} \leftrightarrows \mathcal{T}: F_{*}$ be a pair of adjoint triangulated functors between $t$-categories $\mathcal{S}, \mathcal{T}$ with hearts $\mathcal{A}, \mathcal{B}$. Then $F^{*}$ is right $t$-exact (i.e. $F^{*}(\mathcal{S} \leq 0) \subseteq \mathcal{T}^{\leq 0}$ ) if and only if $F_{*}$ is left exact (i.e. $\left.F_{*}\left(\mathcal{T}^{\geq 0}\right) \subseteq \mathcal{S}^{\geq 0}\right)$. In this case, the functor ${ }^{p} F^{*}: \mathcal{A} \ni A \mapsto H^{0} F^{*}(A) \in$ $\mathcal{B}$ is right exact, ${ }^{p} F_{*}: \mathcal{B} \ni B \mapsto H^{0} F_{*}(B) \in \mathcal{A}$ is left exact and ${ }^{p} F^{*}: \mathcal{A} \leftrightarrows \mathcal{B}:{ }^{p} F_{*}$ form a pair of adjoint functors 
We shall also need this lemma in the proof of Theorem A.4.2:

A.3.2. Lemma. Let $F: \mathcal{S} \rightarrow \mathcal{T}$ be a right exact $t$-functor between $t$ categories $\mathcal{S}, \mathcal{T}$ with hearts $\mathcal{A}, \mathcal{B}$. Assume that ${ }^{p} F: \mathcal{A} \rightarrow \mathcal{B}$ has kernel 0 and that the $t$-structure of $\mathcal{S}$ is non-degenerate. Then $F$ is conservative in the following two cases:

(i) F is t-exact;

(ii) the t-structure of $\mathcal{S}$ is bounded above.

Proof. Let $X \in \mathcal{S}$ be such that $F(X)=0$ : we must show that $X=0$. In case (i), we just use the isomorphism ${ }^{p} H^{i}(F(X))={ }^{p} F\left({ }^{p} H^{i}(X)\right)$ for any $i \in \mathbf{Z}$. In case (ii), let $i$ be an integer such that ${ }^{p} H^{j}(X)=0$ for $j>i$. By right exactness,

$$
0={ }^{p} H^{i}(F(X))={ }^{p} F\left({ }^{p} H^{i}(X)\right)
$$

hence ${ }^{p} H^{i}(X)=0$ and we conclude.

A.4. The homotopy category of an additive category. Throughout this section, $\mathcal{A}$ is an essentially small additive category and $\operatorname{Mod}-\mathcal{A}$ is the category of right $\mathcal{A}$-modules (see $\S 1.2$ ).

The following derived analogue of Proposition 1.2.1 is a special case of a theorem of Bernhard Keller (see [33, Remark 5.3 (a)]).

A.4.1. Proposition. The functor

$$
K^{b}(\mathcal{A}) \stackrel{\iota \mathcal{A}}{\longrightarrow} D(\operatorname{Mod}-\mathcal{A})
$$

induced by the Yoneda embedding is fully faithful, has dense image and identifies $K^{b}(\mathcal{A})^{\natural}$ with the full subcategory of compact objects of $D(\operatorname{Mod}-\mathcal{A})$.

(For a self-contained proof, see the first version of this paper at https://arxiv.org/abs/1506.08385.)

Note that $D(\operatorname{Mod}-\mathcal{A})$ is pseudo-abelian since it has representable infinite direct sums, which justifies the last assertion. Also, by BalmerSchlichting [2], the categories $K^{b}(\mathcal{A})^{\natural}$ and $K^{b}\left(\mathcal{A}^{\natural}\right)$ are equivalent, although we shall not use it.

We now go back to the results of $\S$ A.2. By Proposition A.4.1, the category $\mathcal{S}=D(\operatorname{Mod}-\mathcal{A})$ verifies the hypotheses of Theorem A.2.2, with $\mathcal{S}^{c}=K^{b}(\mathcal{A})^{\natural}$. Thus, if $R$ is a set of objects of $K^{b}(\mathcal{A}), \mathcal{R}=\langle R\rangle \subseteq$ $K^{b}(\mathcal{A})$ and $\mathcal{R}^{\oplus}=\langle y(R)\rangle^{\oplus} \subseteq D(\operatorname{Mod}-\mathcal{A})$, the conclusions of Theorems A.2.2 and A.2.6 apply. So:

(1) $\mathcal{R}^{c}=\mathcal{R}^{\natural}$ and $\left(D(\operatorname{Mod}-\mathcal{A}) / \mathcal{R}^{\oplus}\right)^{c}=\left(K^{b}(\mathcal{A}) / \mathcal{R}\right)^{\natural}$; in particular, $K^{b}(\mathcal{A}) / \mathcal{R} \rightarrow D(\operatorname{Mod}-\mathcal{A}) / \mathcal{R}^{\oplus}$ is fully faithful and dense. 
(2) The projection functor $D(\operatorname{Mod}-\mathcal{A}) \rightarrow D(\operatorname{Mod}-\mathcal{A}) / \mathcal{R}^{\oplus}$ has a right adjoint, which itself has a right adjoint.

Let now $S$ be a set of morphisms in $\mathcal{A}$ which contains all identities and is stable under direct sums. By [29, Th. A.3.3], the category $\mathcal{B}=$ $S^{-1} \mathcal{A}$ is additive, as well as the localisation functor $Q: \mathcal{A} \rightarrow \mathcal{B}$. Thus the setting of $\S 1.2$ applies, and the functor $Q^{*}: \operatorname{Mod}-\mathcal{B} \rightarrow \operatorname{Mod}-\mathcal{A}$ is fully faithful. We may identify $S$ to a set of morphisms in $K^{b}(\mathcal{A})$ via the natural embedding $\eta: \mathcal{A} \ni A \mapsto A[0] \in K^{b}(\mathcal{A})$. If we take for $R=R_{S}$ the set of cones of $\eta(s)$ for $s \in S$, it is natural to ask about the relationship between the above localisations and the categories $K^{b}(\mathcal{B})$, $D(\operatorname{Mod}-\mathcal{B})$. The answer is given by the following theorem.

A.4.2. Theorem. Let $R_{S}$ be as above, and write $\mathcal{R}_{S}, \mathcal{R}_{S}^{\oplus}$ for the corresponding triangulated subcategories of $K^{b}(\mathcal{A})$ and $D(\operatorname{Mod}-\mathcal{A})$.

a) The functor $K^{b}(Q): K^{b}(\mathcal{A}) \rightarrow K^{b}(\mathcal{B})$ factors through $K^{b}(\mathcal{A}) / \mathcal{R}_{S}$.

The functor $L Q_{!}: D(\operatorname{Mod}-\mathcal{A}) \rightarrow D(\operatorname{Mod}-\mathcal{B})$ factors through $D(\operatorname{Mod}-\mathcal{A}) / \mathcal{R}_{S}^{\oplus}$. This yields a naturally commutative diagram

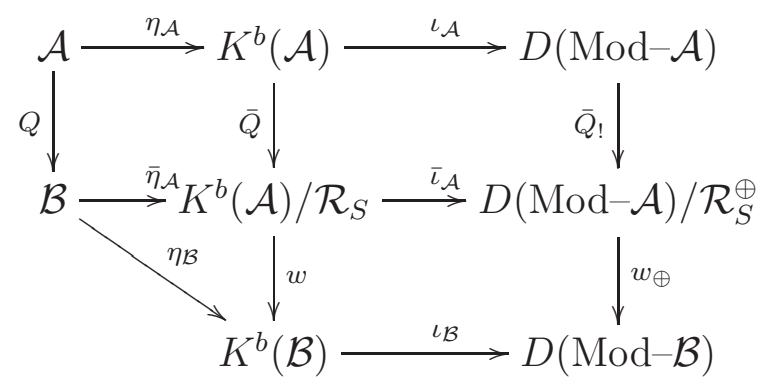

in which all functors not starting from $\mathcal{A}$ or $\mathcal{B}$ are triangulated.

b) The functor $\bar{\iota}_{\mathcal{A}}$ is fully faithful, has dense image, and identifies

$\left(K^{b}(\mathcal{A}) / \mathcal{R}_{S}\right)^{\natural}$ with the full subcategory of compact objects of $D(\operatorname{Mod}-\mathcal{A}) / \mathcal{R}_{S}^{\oplus}$.

c) The functor $\bar{Q}$ ! has a (fully faithful) right adjoint $\bar{Q}^{*}$, which itself has a right adjoint $\bar{Q}_{*}$. The essential image of $\bar{Q}^{*}$ is $D_{\operatorname{Mod}-\mathcal{B}}(\operatorname{Mod}-\mathcal{A})$ (see (3.3)), where $\operatorname{Mod}-\mathcal{B}$ is embedded in $\operatorname{Mod}-\mathcal{A}$ by means of $Q^{*}$.

d) Via $\bar{Q}^{*}$, the natural t-structure of $D(\operatorname{Mod}-\mathcal{A})$ induces a t-structure on $D(\operatorname{Mod}-\mathcal{A}) / \mathcal{R}_{S}^{\oplus}$, with heart $\operatorname{Mod}-\mathcal{B}$; the functor $\bar{Q}_{!}$(resp. $\bar{Q}_{*}$ ) is right (resp. left) t-exact.

e) The functor $\bar{\eta}_{\mathcal{A}}$ is fully faithful; for $B_{1}, B_{2} \in \mathcal{B}$ and $M \in \operatorname{Mod}-\mathcal{B}$, we have

$$
\begin{aligned}
\left(K^{b}(\mathcal{A}) / \mathcal{R}_{S}\right)\left(\bar{\eta}_{\mathcal{A}}\left(B_{1}\right), M[i]\right)=0 & \text { for } i \neq 0 \\
\left(K^{b}(\mathcal{A}) / \mathcal{R}_{S}\right)\left(\bar{\eta}_{\mathcal{A}}\left(B_{1}\right), \bar{\eta}_{\mathcal{A}}\left(B_{2}\right)[i]\right)=0 & \text { for } i>0 .
\end{aligned}
$$

f) The obvious functor

$$
\varphi: D(\operatorname{Mod}-\mathcal{B}) \rightarrow D_{\mathrm{Mod}-\mathcal{B}}(\operatorname{Mod}-\mathcal{A}) \simeq D(\operatorname{Mod}-\mathcal{A}) / \mathcal{R}_{S}^{\oplus}
$$


is right adjoint to $w_{\oplus}$, $t$-exact and conservative; it induces the identity on the hearts.

g) The functor $w_{\oplus}$ is right t-exact and induces the identity on the hearts; its restriction to $D^{-}(\operatorname{Mod}-\mathcal{A}) / \mathcal{R}_{S}^{\oplus}$ is conservative. The functor $w$ is conservative as well.

h) If $Q_{!}: \operatorname{Mod}-\mathcal{A} \rightarrow \operatorname{Mod}-\mathcal{B}$ is exact, $w_{\oplus}$ is an equivalence of categories, and so is $w$ after pseudo-abelian completions.

In part a) if this theorem, note that the total left derived functor $L Q$ ! exists, e.g. by [32, Th. 14.4.3].

Proof. a) is obvious since $S$ gets inverted in $K^{b}(\mathcal{B})$ and $L Q ! y(A)[0]=$ $y(Q(A))[0]$ for $A \in \mathcal{A}$. b) and c) only repeat the points (1) and (2) above, except for the description of the image of $\bar{Q}^{*}$.

Let $C \in D(\operatorname{Mod}-\mathcal{A})$ : by definition, $C \in \operatorname{Im} \bar{Q}^{*}$ if and only if the $\operatorname{map} D(\operatorname{Mod}-\mathcal{A})(y(B)[i], C) \stackrel{s^{*}}{\longrightarrow} D(\operatorname{Mod}-\mathcal{A})(y(A)[i], C)$ is an isomorphism for any $s \in S, s: A \rightarrow B$, and any $i \in \mathbf{Z}$. Since $y(A)$ and $y(B)$ are projective in $\operatorname{Mod}-\mathcal{A}$, this isomorphism may be rewritten: $H_{i}(C)(B) \stackrel{\sim}{\longrightarrow} H_{i}(C)(A)$. Thus $C \in \operatorname{Im} \bar{Q}^{*} \Longleftrightarrow H_{i}(C) \in \operatorname{Im} Q^{*}$ for all $i \in \mathbf{Z}$. d) follows from c) via Lemma A.3.1.

In e), since $Q: \mathcal{A} \rightarrow \mathcal{B}$ is essentially surjective we may write $B_{i} \simeq Q\left(A_{i}\right)$ for $A_{1}, A_{2} \in \mathcal{A}$. Using the full faithfulness of $\bar{\iota}_{\mathcal{A}}$, the first vanishing then follows from adjunction and the projectivity of $y\left(A_{1}\right)$, and the second one follows from the first and the right $t$-exactness of $\bar{Q}_{!}$. It remains to prove the full faithfulness of $\bar{\eta}_{\mathcal{A}}$ : we have

$$
\begin{aligned}
&\left(K^{b}(\mathcal{A}) / \mathcal{R}_{S}\right)\left(\bar{\eta}_{\mathcal{A}}\left(Q\left(A_{1}\right)\right),\right.\left.\bar{\eta}_{\mathcal{A}}\left(Q\left(A_{2}\right)\right)\right) \stackrel{\sim}{\longrightarrow} \\
&\left(D(\operatorname{Mod}-\mathcal{A}) / \mathcal{R}_{S}^{\oplus}\right)\left(\bar{Q}_{!} y\left(A_{1}\right)[0], \bar{Q}_{!} y\left(A_{2}\right)[0]\right) \\
& \simeq D(\operatorname{Mod}-\mathcal{A})\left(y\left(A_{1}\right)[0], \bar{Q}^{*} \bar{Q} ! y\left(A_{2}\right)[0]\right) \\
& \simeq H^{0}\left(\bar{Q}^{*} \bar{Q}_{!} y\left(A_{2}\right)\right)\left(A_{1}\right)=Q^{*} Q_{!} y\left(A_{2}\right)\left(A_{1}\right) .
\end{aligned}
$$

Here we used again the right $t$-exactness of $\bar{Q}_{!}$, plus Lemma A.3.1. But we have

$$
\begin{aligned}
& Q^{*} Q ! y\left(A_{2}\right)\left(A_{1}\right)=Q ! y\left(A_{2}\right)\left(Q\left(A_{1}\right)\right) \\
&=y\left(Q\left(A_{2}\right)\left(Q\left(A_{1}\right)\right)=\mathcal{B}\left(Q\left(A_{1}\right), Q\left(A_{2}\right)\right)\right.
\end{aligned}
$$

which concludes the proof.

f) follows from the adjunction $\left(\bar{Q}_{!}, \bar{Q}^{*}\right)$, the adjunction $\left(L Q_{!}, R Q^{*}\right)$ and the dual of Proposition 3.3.6 a); conservativity follows from Lemma A.3.2 (i). 
In $\mathrm{g}$ ), the first two assertions follow from $\mathrm{f}$ ) in view of Lemma A.3.1. The next claim is a special case of Lemma A.3.2 (ii). The conservativity of $w$ now follows from the full faithfulness of $\iota_{\mathcal{B}}$.

Let us prove $\mathrm{h}$ ). In view of $\mathrm{f}$ ), to show that $w_{\oplus}$ is an equivalence of categories it suffices to show that so is $\varphi$. Since $Q$ ! and $Q^{*}$ are exact, the identity

$$
L\left(Q_{!} Q^{*}\right) \simeq L Q_{!} L Q^{*}
$$

holds trivially in $D(\operatorname{Mod}-\mathcal{B})$. In particular, the counit map $L Q_{!} L Q^{*} \Rightarrow$ $I d$ is an isomorphism and $L Q^{*}$ is fully faithful. Since $\bar{Q}^{*}$ is also fully faithful, we find that $\varphi$ is fully faithful. Since it clearly commutes with infinite direct sums, its essential image $\mathcal{I}$ is localising and to prove the essential surjectivity of $\varphi$ it remains to show that $\mathcal{I}$ is dense. Using b), we reduce to prove that $\mathcal{I}$ contains the image of $\bar{\iota}_{\mathcal{A}} \bar{\eta}_{\mathcal{A}}$.

Let $B \in \mathcal{B}$ and $A \in \mathcal{A}$ such that $B=Q(A)$. Then $\bar{\iota}_{\mathcal{A}} \bar{\eta}_{\mathcal{A}}(B)=$ $\bar{Q}_{!} y(A)[0]$, while $\varphi \iota_{\mathcal{B}} \eta_{\mathcal{B}}(B)=L Q^{*} y(B)[0]=L Q^{*} L Q_{!} y(A)[0]$. We must show that the cone of the counit map $L Q^{*} L Q ! y(A)[0] \rightarrow y(A)[0]$ belongs to $\mathcal{R}_{S}^{\oplus}$, or equivalently that it is left orthogonal to $\mathcal{I}$. It suffices to show that it is left orthogonal to $L Q^{*} M[i]$ for any $M \in \operatorname{Mod}-\mathcal{B}$ and any $i \in \mathbf{Z}$. This follows easily from the full faithfulness of $L Q^{*}$.

The claim for $w$ now follows, since the equivalence $w_{\oplus}$ induces an equivalence between the subcategories of compact objects (see b) and Proposition A.4.1).

A.4.3. Remarks. 1) One can show that, in h), $Q_{\text {! }}$ is exact provided $S$ admits a calculus of right fractions (compare [16, I.3, Prop. 1.1]).

2) In the terminology of Bondarko [8, Def. 4.3.1 1], Theorem A.4.2

e) says that the full subcategory $\mathcal{B} \stackrel{\bar{\eta}_{\mathcal{A}}}{\longrightarrow} K^{b}(\mathcal{A}) / \mathcal{R}_{S}^{o}$ is negative. Since $\bar{\eta}_{\mathcal{A}}(\mathcal{B})$ generates $K^{b}(\mathcal{A}) / \mathcal{R}_{S}^{o}$, the latter carries a weight structure with heart $\bar{\eta}_{\mathcal{A}}(\mathcal{B})^{\natural}$ by loc. cit., Th. 4.3.2. One can then check that the functor $w$ coincides with the weight complex functor $t$ of loc. cit., Th. 3.3.1, which is conservative by part $\mathrm{V}$ of the latter theorem. Theorem A.4.2 provides an alternative proof of this conservativity, using the natural $t$-structure of $D(\operatorname{Mod}-\mathcal{A}) / \mathcal{R}_{S}$ : this seems related to Bondarko's notion of adjacence between a weight structure and a $t$-structure $[8$, §4.4]. In [9, Th. 4.2.2], Bondarko and Sosnilo give a direct proof of Theorem A.4.2 e), without using the full embedding $K^{b}(\mathcal{A}) / \mathcal{R}_{S} \hookrightarrow$ $D(\operatorname{Mod}-\mathcal{A}) / \mathcal{R}_{S}^{\oplus}$.

A.4.4. Example. Let $\mathcal{X}$ be an additive subcategory of $\mathcal{A}$ and let $\mathcal{I}_{\mathcal{X}}$ be the ideal of morphisms in $\mathcal{A}$ which factor through an object of $\mathcal{X}$, compare [1, Ex. 1.3.1]: the projection functor $Q: \mathcal{A} \rightarrow \mathcal{A} / \mathcal{I}_{\mathcal{X}}$ is universal among additive functors mapping all objects of $\mathcal{X}$ to 0 . Then 
$Q$ is a localisation. Indeed, let

$$
S_{\mathcal{X}}=\left\{s \in A r(\mathcal{A}) \mid s \text { becomes invertible in } \mathcal{A} / \mathcal{I}_{\mathcal{X}}\right\} .
$$

Since $S_{\mathcal{X}}$ contains all identities and is stable under direct sums, the localisation $S_{\mathcal{X}}^{-1} \mathcal{A}$ is additive [29, Th. A.3.3]; to show that the natural functor $S_{\mathcal{X}}^{-1} \mathcal{A} \rightarrow \mathcal{A} / \mathcal{I}_{\mathcal{X}}$ is an equivalence of categories, it suffices to show that any object $X \in \mathcal{X}$ maps to 0 in $S_{\mathcal{X}}^{-1} \mathcal{A}$. Let $s: 0 \rightarrow X$ and $t: X \rightarrow 0$ be the canonical maps. Then st and $t s$ both become invertible in $\mathcal{A} / \mathcal{I}_{X}$, hence $s \in S_{\mathcal{X}}$.

\section{REFERENCES}

[1] Y. André, B. Kahn Nilpotence, radicaux et structures monoïdales (with an appendix of P. O'Sullivan), Rend. Sem. Mat. Univ. Padova 108 (2002), 107-291.

[2] P. Balmer, M. Schlichting Idempotent completion of triangulated categories, J. Algebra 236 (2001), 819-834.

[3] L. Barbieri-Viale, B. Kahn On the derived category of 1-motives, Astérisque 381, 2016.

[4] A. Beilinson Remarks on n-motives and correspondences at the generic point, in Motives, Polylogarithms and Hodge Theory, F. Bogomolov and L. Katzarkov, eds., International Press, 2002, 33-44.

[5] A. Beilinson, J. Bernstein, P. Deligne Faisceaux pervers, Astérisque 100, 1984.

[6] A. Beilinson, V. Vologodsky A DG guide to Voevodsky's motives, GAFA (Geom. Funct. Anal.) 17 (2008), 1709-1787.

[7] M. Bökstedt, A. Neeman Homotopy limits in triangulated categories, Compositio Math. 86 (1993), 209-234.

[8] M. Bondarko Weight structures vs. t-structures; weight filtrations, spectral sequences, and complexes (for motives and in general), J. K-theory 6 (2010), 387-504.

[9] M. Bondarko, V. Sosnilo Non-commutative localizations of additive categories and weight structures; applications to birational motives, http: //arxiv.org/abs/1304.6059.

[10] J.-L. Colliot-Thélène Unramified cohomology, birational invariants and the Gersten conjecture, Proc. Symp. pure Math. 58 (I), AMS, 1995, 1-64.

[11] F. Déglise Motifs génériques, Rend. Sem. Mat. Univ. Padova 119 (2008), $173-244$.

[12] F. Déglise Modules homotopiques, Doc. Math. 16 (2011), 411-455.

[13] E. Friedlander, V. Voevodsky Bivariant cycle cohomology, in E. Friedlander, A. Suslin, V. Voevodsky Cycles, transfers and motivic cohomology theories, Ann. Math. Studies 143, Princeton University Press, 2000, $138-187$.

[14] W. Fulton Intersection theory, Springer, 1984.

[15] L. Illusie, Y. Laszlo and F. Orgogozo (avec la collaboration de F. Déglise, A. Moreau, V. Pilloni, M. Raynaud, J. Riou, B. Stroh, M. Temkin et W. 
Zheng), Travaux de Gabber sur l'uniformisation locale et la cohomologie étale des schémas quasi-excellents. Séminaire à l'École polytechnique 2006-2008, Astérisque 363-364 (2014), xxiv+619 pages.

[16] P. Gabriel, M. Zisman Calculus of fractions and homotopy theory, Springer, 1967.

[17] R. Godement Topologie algébrique et théorie des faisceaux, Hermann, 1959.

[18] R. Hartshorne Residues and Duality, Lect. Notes in Math. 20, Springer, 1966.

[19] A. Huber Slice filtration on motives and the Hodge conjecture (with an appendix by J. Ayoub), Math. Nachr. 281 (2008), 1764-1776.

[20] A. Huber, B. Kahn The slice filtration and mixed Tate motives, Compositio Math. 142 (2006), 907-936.

[21] B. Kahn The Geisser-Levine method revisited and algebraic cycles over a finite field, Math. Ann. 324 (2002), 581-617.

[22] B. Kahn Zeta functions and motives, Pure appl. Math. Quarterly 5 (2009) 507-570 [2008].

[23] B. Kahn Relatively unramified elements in cycle modules, J. K-theory 7 (2011), 409-427.

[24] B. Kahn, M. Levine Motives of Azumaya algebras, J. Inst. Math. Jussieu 9 (2010), 481-599.

[25] B. Kahn, J. P. Murre, C. Pedrini On the transcendental part of the motive of a surface, in Algebraic cycles and motives, Part II, LMS Series 344, Cambridge University Press, 2007, 143-202.

[26] B. Kahn, R. Sujatha Birational motives, I (preliminary version), preprint, 2002, http://www.math.uiuc.edu/K-theory/0596/.

[27] B. Kahn, R. Sujatha A few localisation theorems, Homology, Homotopy Appl. 9 (2007), 137-161.

[28] B. Kahn, R. Sujatha Birational geometry and localisation of categories, Doc. Math. - Extra Volume Merkurjev (2015), 167-224.

[29] B. Kahn, R. Sujatha Birational motives, I: pure birational motives, to appear in Annals of $K$-theory.

[30] B. Kahn, R. Sujatha The derived functors of unramified cohomology, preprint, https://arxiv.org/abs/1511.07072.

[31] B. Kahn, T. Yamazaki Voevodsky's motives and Weil reciprocity, Duke Math. J. 162 (14) (2013), 2751-2796.

[32] M. Kashiwara, P. Schapira Categories and sheaves, Grundl. der Math. Wiss. 332, Springer, 2006.

[33] B. Keller Deriving DG categories, Ann. Scient. Éc. Norm. Sup. 27 (1994), 63-102.

[34] H. Knaf, F.-V. Kuhlmann Abhyankar places admit local uniformization in any characteristic, Ann. Sci. Éc. Norm. Sup. 38 (2005), 833-846.

[35] M. Levine The indecomposable $K_{3}$ of fields, Ann. Sci. 'c. Norm. Sup. 22 (1989), 255-344.

[36] C. Mazza, V. Voevodsky, C. Weibel Lecture notes on Motivic cohomology, Clay Math. Monographs 2, AMS, 2006.

[37] A.S. Merkurjev Unramified elements in cycle modules, J. London Math. Soc. 78 (2008) 51-64. 
[38] A. Merkurjev, A. Suslin The group $K_{3}$ for a field (Russian), Izv. Akad. Nauk SSSR 54 (1990), 522-545; translation in Math. USSR-Izv. 36 (1991), 541-565.

[39] J.S. Milne Abelian varieties, Ch. V of Arithmetic Geometry (G. Cornell, J. Silverman, eds.), Springer, 1986, 103-150.

[40] F. Morel, V. Voevodsky $\mathbf{A}^{1}$-homotopy theory of schemes, Publ. Math. IHÉS 90 (1999), 45-143.

[41] A. Neeman The connection between the $K$-theory localisation theorem of Thomason-Trobaugh and Yao and the smashing subcategories of Bousfield and Ravenel, Ann. Sci. Éc. Norm. Sup. 25 (1992), 547-566.

[42] A. Neeman Triangulated categories, Ann. Math. Studies 148, Princeton University Press, 2001.

[43] D. Pauksztelllo Compact cochain objects in triangulated categories and co-t-structures, Centr. Eur. J. of Math. 6 (2008), 25-42.

[44] J. Riou Théorie homotopique des $S$-schémas, mémoire de DEA, Paris 7 , 2002, http://www.math.u-psud.fr/ riou/dea/dea.pdf.

[45] J. Roberts Chow's moving lemma, Appendix 2 to Motives by Steven L. Kleiman, Algebraic geometry, Oslo 1970 (Proc. Fifth Nordic Summer School in Math.), 89-96. Wolters-Noordhoff, Groningen, 1972.

[46] M. Rost Chow groups with coefficients, Doc. Math. 1 (1996), 319-393.

[47] N. Spaltenstein Resolutions of unbounded complexes, Compositio Math. 65 (1988), 121-154.

[48] M. Spieß, T. Szamuely On the Albanese map for smooth quasi-projective varieties, Math. Ann. 325 (2003), 1-17.

[49] M. Somekawa On Milnor K-groups attached to semi-abelian varieties, $K$-theory 4 (1990), 105-119.

[50] A. Suslin, V. Voevodsky Singular homology of abstract algebraic varieties, Invent. Math. 123 (1996), 61-94.

[51] J.-L. Verdier Des catégories dérivées des catégories abéliennes, Astérisque 239, 1996.

[52] V. Voevodsky Cohomological theory of presheaves with transfers, in E. Friedlander, A. Suslin, V. Voevodsky Cycles, transfers and motivic cohomology theories, Ann. Math. Studies 143, Princeton University Press, 2000, 188-187.

[53] V. Voevodsky Triangulated categories of motives over a field, in E. Friedlander, A. Suslin, V. Voevodsky Cycles, transfers and motivic cohomology theories, Ann. Math. Studies 143, Princeton University Press, 2000, $188-238$.

[54] V. Voevodsky Open problems in the motivic stable homotopy theory, I, preprint, 2000.

[55] V. Voevodsky Cancellation theorem, Doc. Math. 2010, Extra volume: Andrei A. Suslin sixtieth birthday, 671-685.

[56] O. Zariski, P. Samuel Commutative Algebra, vol. II, van Nostrand/Springer, 1960/1975.

[SGA4-I] E. Artin, A. Grothendieck, J.-L. Verdier Théorie des topos et cohomologie étale des schémas (SGA4), Vol. 1, Lect. Notes in Math. 269, Springer, 1972 . 
IMJ-PRG, 4 Place Jussieu, 75010 Paris, France

E-mail address: bruno.kahn@imj-prg.fr

University of British Columbia, Vancouver, BC V6T1Z2, Canada

E-mail address: sujatha@math.ubc.ca 